\title{
Pigment Epithelium-Derived Factor Promotes Axon Regeneration and Functional Recovery After Spinal Cord Injury
}

\author{
Andrew R. Stevens ${ }^{1} \cdot$ Umar Ahmed $^{2} \cdot$ Vasanthy Vigneswara $^{1} \cdot$ Zubair Ahmed $^{1}$ (D)
}

Received: 4 January 2019 / Accepted: 15 April 2019 / Published online: 2 May 2019

(C) The Author(s) 2019

\begin{abstract}
Although neurons in the adult mammalian CNS are inherently incapable of regeneration after injury, we previously showed that exogenous delivery of pigment epithelium-derived factor (PEDF), a 50-kDa neurotrophic factor (NTF), promoted adult retinal ganglion cell neuroprotection and axon regeneration. Here, we show that PEDF and other elements of the PEDF pathway are highly upregulated in dorsal root ganglion neurons (DRGN) from regenerating dorsal column (DC) injury paradigms when compared with non-regenerating DC injury models. Exogenous PEDF was neuroprotective to adult DRGN and disinhibited neurite outgrowth, whilst overexpression of PEDF after DC injury in vivo promoted significant DC axon regeneration with enhanced electrophysiological, sensory, and locomotor function. Our findings reveal that PEDF is a novel NTF for adult DRGN and may represent a therapeutically useful factor to promote functional recovery after spinal cord injury.
\end{abstract}

Keywords PEDF $\cdot$ Spinal cord injury $\cdot$ Serum withdrawal $\cdot$ Dorsal root ganglia neurons $\cdot$ CNS $\cdot$ Axon regeneration $\cdot$ Neurite outgrowth

\section{Introduction}

Injury to the central nervous system (CNS) is a considerable cause of morbidity and mortality in modern populations. The intrinsic inability of the CNS to repair on a cellular level leads to poor outcomes for rehabilitation on a patient level. Neuronal cells lost through injury are not replaced, and axons do not regenerate; the damage acquired causes a permanent functional deficit. Whilst spinal cord injury (SCI) presents a pertinent clinical problem, no regenerative therapy is available which offers an adequate level of functional recovery [1]. Pigment epithelium-derived factor (PEDF) is a 50-kDa glycoprotein and a member of the serine protease inhibitor (serpin) gene

Electronic supplementary material The online version of this article (https://doi.org/10.1007/s12035-019-1614-2) contains supplementary material, which is available to authorized users.

Zubair Ahmed

z.ahmed.1@bham.ac.uk

1 Neuroscience and Ophthalmology, Institute of Inflammation and Ageing, College of Medical and Dental Sciences, Robert Aitken Institute of Clinical Research, University of Birmingham, Birmingham B15 2TT, UK

2 King Edward VI Camp Hill School for Boys, Vicarage Road, Kings Heath, Birmingham B14 7QJ, UK family, with neuroprotective and angiogenic properties. PEDF has been noted for its neuroprotective activity throughout the CNS $[2,3]$. Whilst PEDF has been shown to promote survival and neurite outgrowth of motor neurons, evidence is limited to in vitro studies utilising embryonic avian cultures [4]. More recently PEDF has been shown to be both neuroprotective and axogenic in adult retinal ganglion cells after optic nerve crush injury $[5,6]$. We therefore asked the question as to whether PEDF is neuroprotective and axogenic to spinal neurons.

To test this hypothesis, we utilised dorsal root ganglion neurons (DRGN), whose cell bodies subserve both the central and peripheral projections, yet these neurons can regenerate their peripheral projections but not their central projections. These DRGN can be placed under different in vivo conditions to create regenerating and non-regenerating models of CNS injury [7-11]. A non-regenerating SCI can be modelled by administering a dorsal column (DC) crush injury; DRGN axons barely regenerate but do not extend into the DC injury site. A peripheral regenerating paradigm can be modelled by a sciatic nerve (SN) crush injury; axons will extend into the SN injury site. By combining these models, pre-conditioning (p) $\mathrm{SN}$ lesions, i.e. administering an $\mathrm{SN}$ crush lesion 7 days prior to DC injury ( $\mathrm{pSN}+\mathrm{DC}$ ), the central axons will extend into and through the DC injury site as well as peripheral axons extending into the SN lesion site [8]. Pre-conditioning is explained by upregulation of gene transcription, which enables 
DRGN to enter a 'growth-activated state', rendering them capable of regenerating their axons into the DC lesion site [10-13].

Here, we investigated DRGN survival and regeneration in response to PEDF in the adult spinal cord, evaluating the potential for PEDF to be of value as a regenerative therapy in the clinical setting. We show that PEDF is activated in regenerating models of DC injury, promotes DRGN survival and neurite outgrowth in a serum-withdrawal model in vitro, promotes DC axon regeneration and improved functional recovery in vivo, and causes the release of several Trkdependent neurotrophic factors (NTF) that account for some of its survival/neurite-growth promoting effects.

\section{Methods}

\section{Regenerating and Non-Regenerating DC Injury Models}

All surgical procedures were licenced by the UK Home Office and ethically approved by the University of Birmingham's Animal and Ethical Review Board. Surgeries were carried out in strict accordance to the guidelines of the UK Animals Scientific Procedures Act, 1986, and the Revised European Directive 1010/63/EU and conformed to the guidelines and recommendation of the use of animals by the Federation of the European Laboratory Animal Science Associations. Adult female Sprague-Dawley rats (170-220 g) (Charles River, Margate, UK) were randomly allocated to either: (1) Sham (control; partial laminectomy but no DC crush injury); (2) DC crush injury (non-regenerating; partial laminectomy followed by DC crush injury); (3) SN crush injury (regenerating $\mathrm{SN}$ ); and (4) pre-conditioning (p) SN crush injury $(\mathrm{pSN}) 1$ week prior to DC crush injury $(\mathrm{pSN}+\mathrm{DC})$. Where specified intact animals with no surgical procedures were also used. All lesions were administered under 5\% isoflurane-induced inhalation anaesthesia, with $1.5 \mathrm{l} / \mathrm{min} \mathrm{O}_{2}$. Pre- and post-injury analgesia was also provided.

DC crush injury was administered bilaterally at the T8 vertebral level as described by us previously $[9,14]$. Briefly, calibrated watchmaker's forceps were inserted through the dorsal cord meninges to a depth of $1.5 \mathrm{~mm}$, and a DC crush was performed. SN crush was performed on the left $\mathrm{SN}$, at the level of the sacro-tuberous ligament, exposed at mid-thigh level. DC injury with pre-conditioning SN injury models were carried out as described, with SN injury preceding DC injury by 1 week.

All in vivo experiments were performed by investigators masked to the treatment conditions with animals randomly assigned to treatment groups. For the microarray experiments, four independent samples of DRG were from two pooled DRG per experimental group from intact, $\mathrm{DC}, \mathrm{SN}$, and $\mathrm{pSN}$
+ DC animals to ensure enough high-quality RNA could be extracted ( $n=8$ rats/group, total for experiment $=32$ rats). For quantitative RT-PCR (qRT-PCR), RNA from four DRG/group ( $n=4$ rats/group) were extracted to validate highly changed genes in DC, $\mathrm{SN}$, and pSN + DC animals and repeated on three independent occasions ( $n=12$ rats/group, total for experiment $=36$ rats). For immunohistochemistry to determine PEDF and PEDF-R localisation, three DRG/group were used and repeated on three independent occasions ( $n=9$ rats/group, total for experiment $=36$ rats). To determine levels of PEDF by Western blot in L4/5 DRG from intact and after DC, SN and $\mathrm{pSN}+\mathrm{DC}$ injuries, total protein extracts were prepared from $n=3$ rats/group and repeated on three independent occasions ( $n=9$ rats/group, total for experiment $=36$ rats).

To determine if PEDF overexpression by viral vectormediated (adeno-associated vector, serotype 8-AAV8) delivery to DRGN promotes DC axon regeneration and functional recovery (sensory and locomotor), $n=6$ rats/group were used, repeated on three independent occasions, comprising: Sham, $\mathrm{DC}+\mathrm{AAV}-\mathrm{Null}$ (control) and DC + AAV-PEDF (total for experiment $=54$ rats). Rat PEDF was overexpressed in DRGN using AAV8 under the control of a CMV promoter, referred to as AAV-PEDF from herein (AAV8-rat-SERPINF1; cat no. AAV-291714; Vector Biolabs, Malvern, PA, USA). An AAV8-Null vector under the control of a CMV promoter, referred to as AAV-Null from herein (AAV8-Null; Cat no. 7077, Vector Biolabs) was used to control for AAV-PEDF effects in vivo. $1 \times 10^{13}$ viral particles of each vector in a final volume of $5 \mu$ of PBS was injected directly into the DRG (intra-DRG) as described by us previously [15]. Axon regeneration and sensory and locomotor function were assessed at 6 weeks after SCI and treatment, as described later. Electrophysiology ( $n=6$ rats/group, 3 independent repeats, total for experiment $=54$ rats) was also performed at 6 weeks after injury and treatment, as described below.

Since AAV8-mediated overexpression is not translational as it requires 1-2 weeks to reach maximum expression of the transgene, we used in vivo-jetPEI, a non-viral vector, which is just as efficient as AAV8, transducing up to $30 \%$ of DRGN after intra-DRG injection [16]. Therefore, we determined if PEDF overexpression by in vivo-jetPEI (referred to as PEI from herein; Polyplus Transfection, NY, USA) promoted functional recovery after DC injury. We used $n=6$ rats/ groups comprising: Sham, DC + Vehicle, DC + PEI-Null, and DC+PEI-PEDF, repeated on three independent occasions (total for experiment $=72$ rats) to perform electrophysiology, tape sensing, and removal and ladder crossing tests as described below. DC crush, preparation and injection of PEI into L4/L5 DRG, electrophysiology, and functional tests were performed as described by us previously [16]. Rats were killed at either 28 days for qRT-PCR and enzyme-linked immunosorbent assay (ELISA) to determine levels of PEDF overexpression or at 6 weeks to perform electrophysiology [16]. 
All other DRGN culture experiments used a total of 60 rats (4 rats/group, run in duplicate and repeated on 3 independent occasions). Animals were killed by exposure to rising concentrations of $\mathrm{CO}_{2}$.

\section{Microarray Analysis}

Groups of four rats (randomised and identity masked) comprising intact control, DC, SN, and pSN + DC injured animals were treated as described above, and L4/L5 DRG pairs were harvested from each animal and RNA extracted was extracted using TRIzol reagent following the manufacturer's protocol (Invitrogen, Paisley, UK). The quality of extracted RNA was checked using a Bioanalyzer. The rat genome AROSTM V3.0set (Operon Biotechnologies GmbH, Cologne, Germany) containing 26,962 long-mer probes representing 22,012 genes and 27,044 gene transcripts was used for the microarray analysis as described by us previously [17].

\section{RNA Extraction and Quantitative RT-PCR}

Total RNA was extracted from harvested DRG (randomised and identity masked) at appropriate timepoints after injury with or without treatment using TRIzol reagent following the manufacturer's protocol (Invitrogen). For cell culture experiments, total RNA was extracted directly from wells following the manufacturer's instructions. The levels of mRNA of selected genes were determined using pre-validated rat primer sequences from complimentary DNA prepared from extracted mRNA, and qRT-PCR was performed using a LightCycler PCR machine (Roche, Burgess Hill, UK) [18]. Primer sequences included PEDF-Rn00709999_m1; AktRn00583646_m1; p50-Rn00550870_m1; SRFRn01757240_m1; BDNF-Rn02537967_S1; NGFRn01533872_m1; GDNF-Rn00569510_m1; Bcl2Rn99999125_m1 (all from Thermo Fisher Scientific, Leicestershire, UK). Fold changes were computed using the $\Delta \Delta \mathrm{Ct}$ method [18].

\section{Pathway Analysis}

Data from mRNA microarray studies of DRGN models of injury was utilised to analyse all genes in the PEDF pathway. Gene expression for each model was standardised as fold increase compared with intact DRGN. Genes significantly altered (greater or lower than 2-fold) in injury models were represented in a schematic pathway with their corresponding fold changes.

\section{Immunohistochemistry}

After killing animals by exposure to rising concentrations of $\mathrm{CO}_{2}$, rats (randomised and masked) were intracardially perfused with $4 \%$ formaldehyde (TAAB Laboratories, Berkshire, UK) in $0.1 \mathrm{M}$ phosphate-buffered saline (PBS). DRG were removed from vertebral levels L4 and L5, postfixed in $4 \%$ formaldehyde and subjected to a graded series of sucrose solutions for cryoprotection. Tissues were blocked in OCT mounting compound (TAAB laboratories) and sectioned at $15 \mu \mathrm{m}$-thick using a cryostat (Brights Instruments, Huntingdon, UK) before being adhered to charged glass slides (Thermo Fisher Scientific, Loughborough, UK). Slides were kept at $-20{ }^{\circ} \mathrm{C}$ until required. Immunohistochemistry was performed as described by us previously [14]. Briefly, slides were washed in three changes of PBS, followed by three further washes in PBS containing 1\% ( $v / v)$ Triton X-100 (Sigma) (PBS-T) to permeabilise cells. Sections were then blocked for $1 \mathrm{~h}$ at room temperature (RT) using PBS containing $0.05 \%$ ( $w /$ $v$ ) bovine serum albumin (Sigma, Poole, UK) and $0.05 \%$ Tween-20 (Sigma) and incubated overnight (16-18 h) at $4{ }^{\circ} \mathrm{C}$ in a humidified chamber with appropriate primary antibodies: goat anti-PEDF (1:400 dilution, R\&D Systems, Oxford, UK)); mouse anti-NF200 (1:400 dilution, Sigma); and rabbit anti-PEDF-R (1:500 dilution, R\&D Systems). Slides were washed $3 \times$ in PBS-T before incubation for $1 \mathrm{~h}$ at RT with the relevant secondary antibody: Alexa488 antigoat; Texas Red anti-mouse; Alexa594 anti-rabbit, all diluted at 1:400, all from Invitrogen). Slides were washed twice in PBS-T, followed by twice in PBS. Coverslips were then mounted using Vectashield mounting medium (containing DAPI) (Vector Laboratories, Peterborough, UK). Negative controls were included in each run where primary antibodies were omitted, and these slides were used to set the background threshold levels prior to image capture.

\section{Protein Extraction, Western Blot, and Densitometry}

Total protein, Western blot, and subsequent densitometry were performed as described by us previously [5]. Briefly, total protein was extracted from L4/L5 DRG from three rats (randomised and masked; repeated on 3 independent occasions, total $n=9$ rats/group) after DC injury and pooled together to ensure enough protein. Forty micrograms of total protein was resolved on $12 \%$ SDS polyacrylamide gels and blotted onto polyvinylidene fluoride (PVDF) membranes (Millipore, Watford, UK) and probed with relevant primary antibodies: goat anti-PEDF (1:500 dilution, R\&D Systems) and $\beta$-actin (1:1000 dilution, Sigma; protein loading control). Membranes were then incubated with relevant HRP-labelled anti-goat and anti-mouse IgG secondary antibodies and bands were detected using the enhanced chemiluminescence kit (GE Healthcare, Buckinghamshire, UK).

For densitometry, Western blots were scanned into Adobe Photoshop (Adobe Systems Inc., San Jose, CA, USA), and the integrated density of bands was analysed using the built-inmacros for gel analysis in ImageJ (NIH, USA, http://imagej. 
nih.gov/ij) by an investigator masked to the treatment conditions $[5,11,19]$. Means \pm SEM were plotted in Microsoft Excel (Microsoft Corporation, CA, USA).

\section{Neurotrophic Factor Enzyme-Linked Immunosorbent Assay}

The levels of brain-derived neurotrophic factor (BDNF), glialderived neurotrophic factor (GDNF), and nerve growth factor (NGF) in culture were detected by ELISA using the appropriate rat ELISA kits, according to the manufacturer's instructions (Abcam, Cambridge, UK) by an investigator masked to the treatment conditions. A rat PEDF ELISA kit was used to detect PEDF in DRG after intra-DRG injection of AAV-PEDF and after pSN + DC lesions, according to the manufacturer's instruction (Elbascience, Houston, TX, USA). ELISA was performed on $10 \mu \mathrm{l}$ of each sample, in duplicate, and repeated on three independent occasions.

\section{Adult DRGN Primary Cell Cultures}

For primary DRGN cultures, adult (170-220 g) female Sprague-Dawley rats (Charles River) were used as described by us previously [20]. Briefly, DRG pairs (T1-L7) were removed and dissociated into single cells using $0.1 \%$ collagenase (Sigma). DRGN were then centrifuged through a $15 \%$ BSA gradient and the resultant cell pellet was re-suspended in Dulbecco's modified eagle medium (DMEM) in 8-well glass chamber slides (BD Biosciences, Oxford, UK), pre-coated with $100 \mu \mathrm{g} / \mathrm{ml}$ poly-D-lysine and $20 \mu \mathrm{g} / \mathrm{ml}$ laminin (both from Sigma). Dissociated DRGN were plated at 500 cells/ well with (DMEM) containing 1\% penicillin-streptomycin (PS) (all from Invitrogen) and $30 \mu \mathrm{M} \mathrm{5-fluoro-2-}$ deoxyuridine (5-FDU, Sigma) to limit non-neuronal cell proliferation [20].

\section{In Vitro Serum Withdrawal Model}

For the serum withdrawal model, DRGN were plated in $500 \mu \mathrm{l}$ of $\mathrm{DMEM} /$ foetal bovine serum/penicillinstreptomycin in 8 -well chamber slides, as described by us previously [5]. After 20-24-h incubation, the medium was replaced with DMEM: with or without serum, and with or without human recombinant PEDF (Peprotech, London, UK) in the presence of 5-FDU. K252a was used at a final concentration of $50 \mathrm{nM}$ whilst $\mathrm{Trk} / \mathrm{A} / \mathrm{B} / \mathrm{C}-\mathrm{Fc}$ fusion proteins were used at $5 \mu \mathrm{g} / \mathrm{ml}$ each [21]. Chamber slides were incubated for $120 \mathrm{~h}$ before being fixed in $4 \%$ formaldehyde and subsequent immunocytochemistry, as described below. All experiments were performed in duplicate and repeated on four independent occasions ( $n=8$ wells/condition) by an investigator masked to the treatment conditions.

\section{Treatment of DRGN and ELISA to Determine NTF Concentrations}

DRGN were treated in duplicates ( $n=8$ wells/condition) with or without PEDF, culture media was collected after $120 \mathrm{~h}$, and samples were assayed to determine the concentrations of NTF using rat-specific ELISA kits, according to the manufacturer's protocol (R\&D Systems) by an investigator masked to the treatment conditions.

\section{Immunocytochemistry}

Immunocytochemistry was performed as described by us previously [5] by an investigator masked to the treatment conditions. Briefly, cultures were fixed for 10 min using $4 \%$ formaldehyde, followed by $\times 3$ washes in PBS. Non-specific staining was blocked for $10 \mathrm{~min}$ in PBS containing 3\% BSA $(w / v)$ and $1 \%$ Triton $\mathrm{X}-100$, followed by incubation with primary antibody: mouse anti- $\beta$ III-tubulin (to mark DRGN soma and neurites, diluted 1:200) for $1 \mathrm{~h}$ at RT, diluted in PBS with 3\% BSA and $0.5 \%(v / v)$ Tween-20. Plates were washed and incubated with Alexa488-labelled anti-mouse IgG secondary antibody (Invitrogen, 1:400 dilution) for $1 \mathrm{~h}$ before mounting in Vectamount with DAPI (Vector Laboratories).

\section{DRGN Survival and Neurite Outgrowth}

With the experimenter masked to the treatment conditions, mean numbers of $\beta$ III-tubulin ${ }^{+}$DRGN was counted in 9 quadrants/well and the total number of DRGN determined as described before [20]. The number of DRGN with neurites and the mean neurite lengths were also quantified in 9 quadrants/well. The longest neurite was measured using Axiovision (Zeiss) from at least $180 \mathrm{DRGN} /$ condition, whilst total DRGN counts to assess survival were made in all wells.

\section{Microscopy and Image Analysis}

Immunostained sections were viewed under an epifluorescent microscope (Axioplan 200) equipped with an Axiocam HRc and Axiovision software (all from Zeiss, Hertfordshire, UK). For each experiment, exposure times were set to optimise images of control slides and adjusted to account for background staining in negative controls (no primary antibody). An investigator masked to the treatment conditions performed image capture and analysis.

To quantify fluorescence, images were captured at $\times 10$ magnification of the entire DRG section, each taken from the same depth through the DRG, adjusted equally in Photoshop, and merged to a composite image using the Photomerge tool. In ImageJ (National Institutes of Health, USA), six areas of DRGN/section ( $n=12 \mathrm{DRG} /$ condition) were selected and mean pixel counts recorded. 

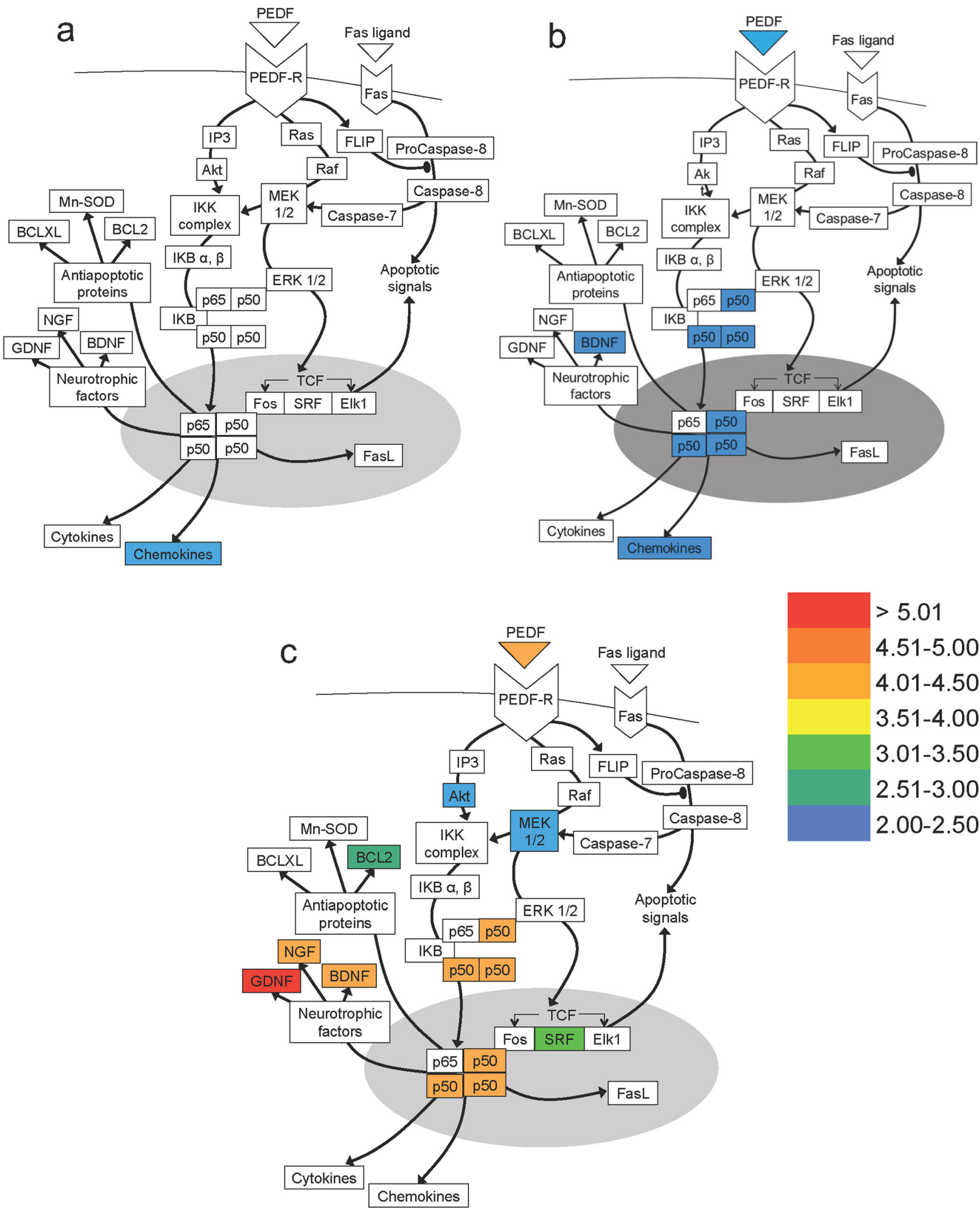

$>5.01$

$4.51-5.00$

$4.01-4.50$

$3.51-4.00$

$3.01-3.50$

$2.51-3.00$

$2.00-2.50$

Fig. 1 Microarray data showing changes in mRNA of molecules of the PEDF pathway in non-regenerating and regenerating DRGN models. Fold difference in mRNA extracted from DRG 10 days post-lesion after a DC, b SN, and $\mathbf{c}$ pSN + DC models. Fold increase in mRNA was normalised to the intact controls. Curved line indicates cell membrane.
Grey oval indicates nucleus. Arrows indicate promoting interaction. Arrows with oval tips indicate inhibitory interactions. Colour chart indicates fold-increase by colour heat with indicated levels of gene expression (fold-changes compared with the intact controls) 
To measure neurite outgrowth, images of DRGN immunostained for $\beta$ III-tubulin were captured for 30 randomly selected DRGN per well. Using Axiovision Software (Carl Zeiss), the number of DRGN with neurites and the length of the longest neurite were recorded from a minimum of $180 \mathrm{DRGN} /$ condition [20]. Image capture and subsequent analyses were performed by an investigator masked to the treatment conditions.

\section{Quantification of Axons}

Axon regeneration in the spinal cord was quantified using GAP43 ${ }^{+}$immunoreactivity, according to previously published methods [22] by an investigator masked to the treatment conditions. GAP43 was used since Cholera toxin B labelling did not work in the rat [22]. Briefly, serial parasagittal sections of cords were reconstructed by collecting all serial $50-\mu \mathrm{m}$-thick sections ( $\sim 70-80$ sections/animal; $n=18$ rats/treatment $)$, and the numbers of intersections of GAP $43^{+}$fibres through a dorsoventral-orientated line were counted by a masked investigator, from $4 \mathrm{~mm}$ rostral to $4 \mathrm{~mm}$ caudal to the lesion site. Finally, axon number was calculated as a percentage of the fibres seen $4 \mathrm{~mm}$ above the lesion, where the DC was intact by an investigator masked to the treatment conditions.

\section{Electrophysiology}

Compound action potentials (CAP) were recorded at 6 weeks after DC injury and treatment, as described previously [16]. Briefly, the experimenter was masked to the treatment status of the animals and the CAP amplitude was calculated between the negative deflection after the stimulus artefact and the next peak of the wave. CAP area was also calculated by rectifying the CAP component (full-wave rectification) and measuring its area. To confirm our recordings and that a CAP could not be recorded, the dorsal half of the spinal cord was transected after each experiment between the stimulating and recording electrodes.

\section{Functional Tests}

Functional testing after DC lesion and treatment was carried out as described previously [16, 23]. Briefly, animals $(n=18 /$ group) were first trained to master traversing a rope and a horizontal ladder for 1 week before functional testing. Baseline parameters were established by performing tests at 2-3 days before injury. Animals were then tested at 2 days, 1 week, 2 weeks, 3 weeks, 4 weeks, 5 weeks, and 6 weeks after DC injury + treatment. Experiments were performed by an observer masked to the treatment conditions in the same order and time of day with each test performed for three individual trials.

Horizontal Ladder Test This tests the animal's locomotor function and is performed on a $0.9-\mathrm{m}$-long horizontal ladder with a diameter of $15.5 \mathrm{~cm}$ and randomly adjusted rungs with
Fig. 2 Confirmation of highly changed genes in pSN + DC DRGN by qRT-PCR and confirmation of changes in PEDF. a Confirmation of highly upregulated levels of PEDF, Akt, MEK1/2, p50, SRF, BDNF, NGF, GDNF, and Bcl 2 by qRT-PCR. b Immunohistochemistry at 10 days after DC injury and $\mathbf{c}$ quantification of immunopositive staining at 1,3, 7, and 15 days post-DC injury showed high levels of PEDF immunoreactivity (green) in regenerating $\mathrm{SN}$ and $\mathrm{pSN}+\mathrm{DC} \mathrm{DRGN}$ soma (red) (DAPI = blue, cell nuclei), whilst low levels were detected in intact and DC-injured DRGN. d Western blot and subsequent densitometry detected high levels of PEDF in regenerating SN and pSN + DC models, with the highest levels of PEDF being present in pSN + DC DRG. e PEDF-R (green) was present in DRGN and remained unchanged in intact and all injury models. DAPI (blue) $=$ cell nuclei. Scale bars in $\mathbf{b}$ and $\mathbf{e}=25 \mu \mathrm{m}$. $*=$ $P<0.05, * *=P<0.001$, ANOVA; $* * *=P<0.0001$, ANOVA

variable gaps of $3.5-5.0 \mathrm{~cm}$. Animals were assessed traversing the ladder, and the left and right rear paw slips were recorded along with the total number of steps and the mean error rate as: the number of slips/total number of steps.

Tape Removal Test (Sensory Function) The tape removal test determines touch perception from the left hind paw. After holding animals with both hind paws extended, the time it took for the animal to detect and remove a piece of tape of $15 \times 15 \mathrm{~mm}$ (Kip Hochkrepp, Bocholt, Germany) affixed to the palm of the left hind paw was recorded and used to calculate the mean sensing time.

\section{Statistical Analysis}

Statistical significance was calculated from sample means using one-way analysis of variance (ANOVA) with post hoc Dunnett's method using SPSS Statistics 19 (IBM, NY, USA).

For the horizontal ladder crossing and tape removal tests, data was analysed as described previously [16, 23] using $\mathrm{R}$ package (www.r-project.org). Briefly, for the ladder crossing test, whole time course of lesioned and Sham-treated animals were compared using binomial generalised linear mixed models (GLMM). Binomial GLMMs were fitted in R using package lme 4 with the glmer function. $P$ values were then calculated using parametric bootstrap. For the tape removal test, linear mixed models (LMM) were calculated by model comparison in $\mathrm{R}$ using the package pbkrtest, with the Kenward-Roger method [16, 23].

\section{Results \\ PEDF Signalling Pathway Is Upregulated in Regenerating Models of DC Injury}

To investigate endogenous genetic changes in nonregenerating $\mathrm{DC}$ and regenerating $\mathrm{SN}$ and $\mathrm{pSN}+\mathrm{DC}$, microarray analysis of mRNA levels in DRGN was performed at 
a

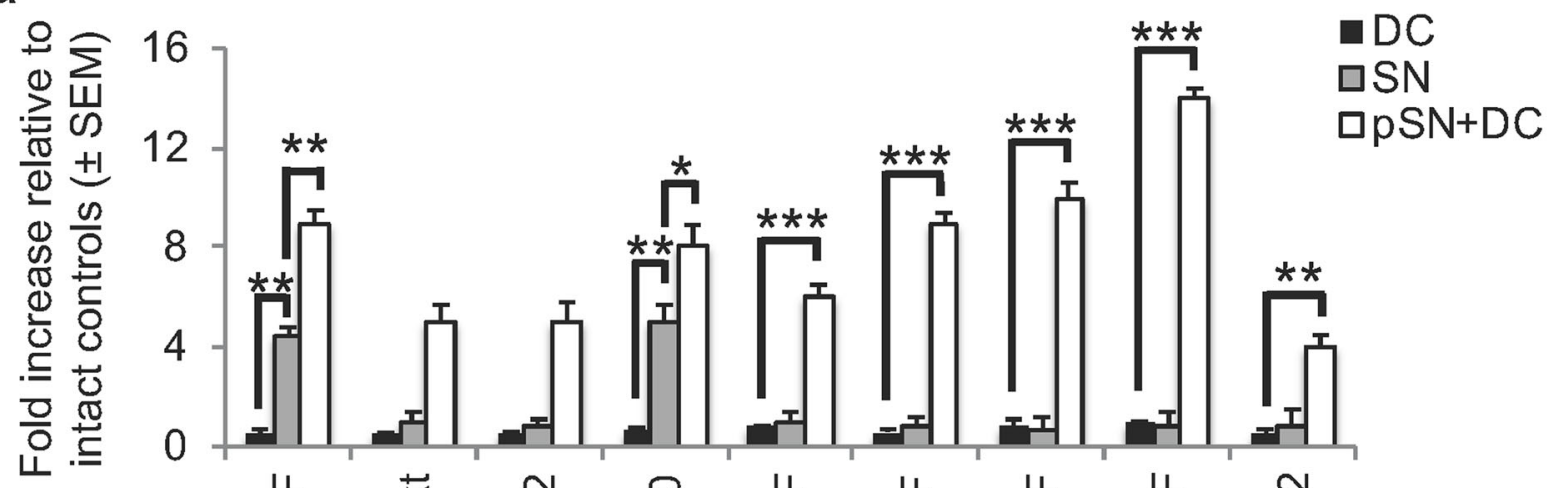

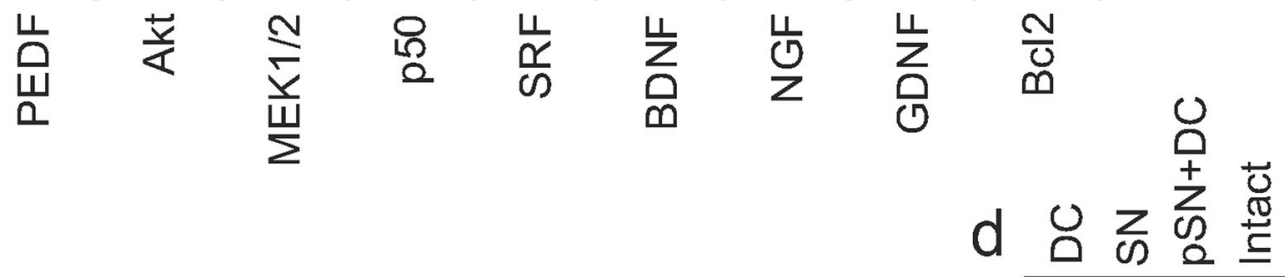
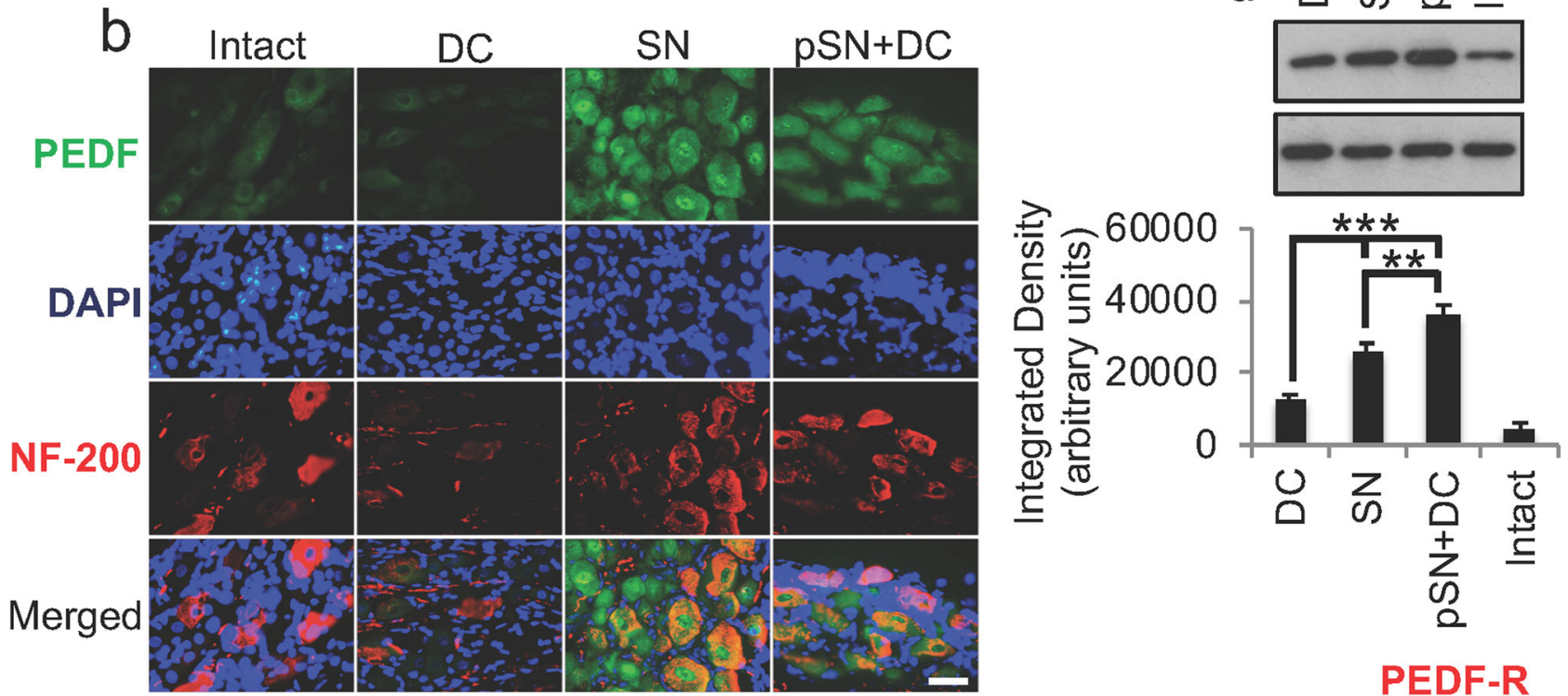

C

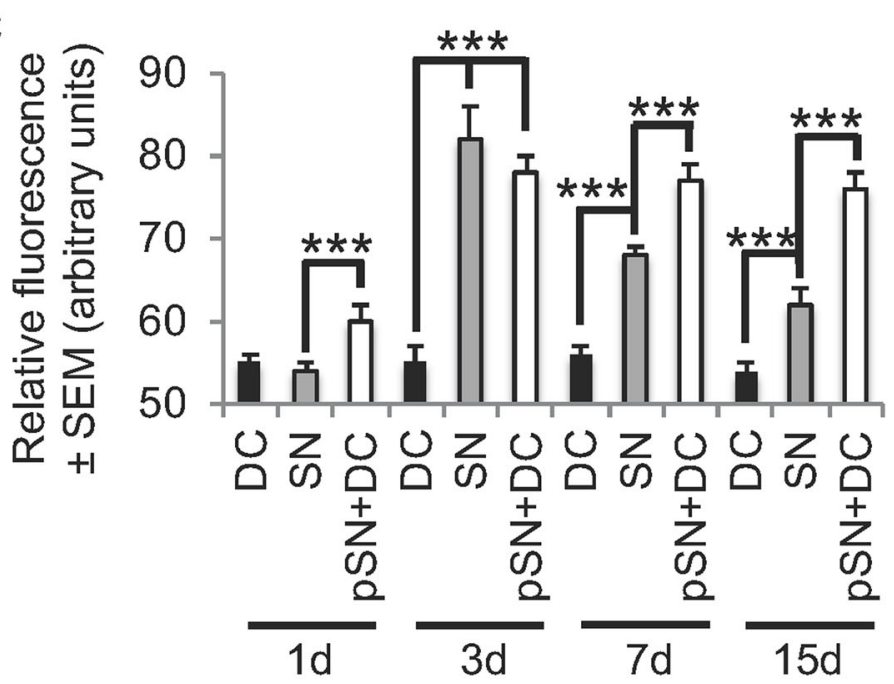

e PEDF-R PEDF-R

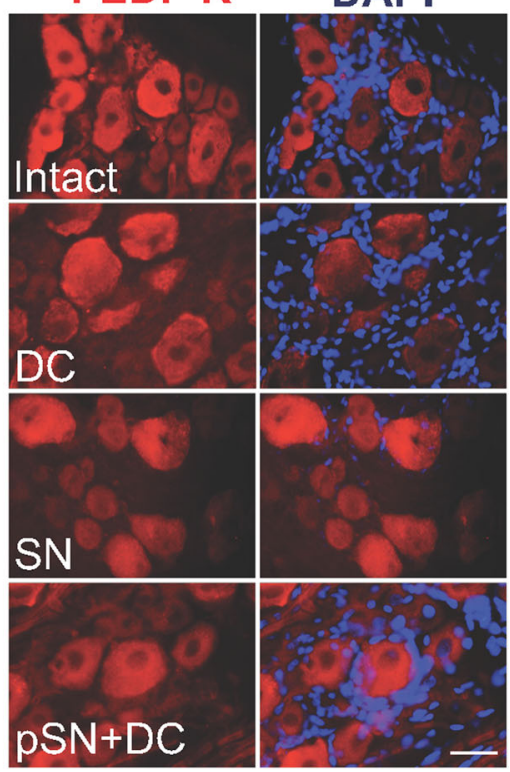


$10 \mathrm{dpl}$. Out of the 156 genes currently known to be involved in the PEDF pathway, 53, 49, and 53 molecules in DC, SN, and pSN + DC models, respectively, showed any mRNA changes compared with the intact controls with all molecules showing significant changes (i.e. $>2$-fold regulation) depicted in Fig. 1. In non-regenerating DC models, only 3 molecules in the PEDF pathway were upregulated > 2-fold: mRNA for chemokines CCL2, CCL5, and CCL6 were upregulated 2.22-, 2.55-, 2.01-fold, respectively (Fig. 1a). In regenerating SN models, 8 molecules changed $>2$-fold, including the transcription factor $\mathrm{NF} \kappa \mathrm{B}$ ( $\mathrm{p} 50$, p65) which were upregulated between 2.00- and 2.50-fold and BDNF was also upregulated by 2.27 -fold (Fig. 1b). The most marked upregulation occurred in the regenerating pSN + DC model where 20 molecules changed $>2$-fold (Fig. 1c). These included mRNA for PEDF, which was 4.33-fold upregulated and transcription factor NFKB (p50, p65) that was upregulated between 4.00- and 4.50 -fold, respectively. Furthermore, there was a 2.55 -fold upregulation of mRNA for anti-apoptotic protein $\mathrm{Bcl} 2$. In addition, three NTF were highly upregulated, including glialderived neurotrophic factor (GDNF; 5.04-fold), nerve growth factor (NGF; 4.32-fold) and brain-derived neurotrophic factor (BDNF; 4.25-fold) (Fig. 1c). These results suggest that PEDF and other pathway molecules were positively correlated with DC axon regeneration.

\section{PEDF mRNA and Protein Are Present in High Levels in Regenerating SN and pSN + DC Paradigms}

To validate the microarray changes observed, we performed qRT-PCR for a selection of the most highly changed genes and showed that the data corroborated our microarray findings. These results showed significant changes in expression of PEDF, Akt, MEK1/2, p50, SRF, BDNF, NGF, GDNF, and $\mathrm{Bcl} 2$ in pSN + DC models compared with SN and DC models (Fig. 2a). To investigate the relationship between PEDF and an enhanced growth-activated state of CNS neurons in vivo, $\mathrm{PEDF}$ and its relative levels were assessed in non-regenerating $\mathrm{DC}$ and regenerating $\mathrm{SN}$ and $\mathrm{pSN}+\mathrm{DC}$ models of injury. PEDF immunostaining (green) was weakly positive in DRGN (red) in the intact control and non-regenerating DC models (Fig. 2b). However, PEDF immunostaining was highly positive in DRGN in regenerating $\mathrm{SN}$ and $\mathrm{pSN}+\mathrm{DC}$ models (Fig. 2b). Interestingly, PEDF immunoreactivity in DRGN from regenerating SN models was localised to the nucleus (arrows) and DRGN somata, whilst in the regenerating $\mathrm{pSN}+\mathrm{DC}$ model, immunoreactivity was mainly localised in DRGN somata.

Quantification of the immunoreactivity for PEDF over time after injury in non-regenerating and regenerating models showed that immunoreactivity reduced over time in regenerating $\mathrm{SN}$ models but remained significantly elevated $(P<0.0001$, ANOVA for DC versus $\mathrm{SN}$ and $\mathrm{pSN}+\mathrm{DC}$ at
Fig. 3 PEDF peptide promotes DRGN survival in a serum withdrawal model. a Approximately 40 and $60 \%$ of DRGN die after 3 and 5 days, respectively, when cultured in the absence of serum. b Supplementation with increasing concentrations of PEDF improved DRGN viability to $85 \%$ with $100 \mathrm{ng} / \mathrm{ml}$ PEDF after 5 days in culture, similar to that observed in the presence of serum. $\mathbf{c}$ Representative images to show DRGN neurite outgrowth after stimulation with $100 \mathrm{ng} / \mathrm{ml}$ PEDF, with and without serum. d DRGN survival and e mean neurite length after treatment with $100 \mathrm{ng} / \mathrm{ml}$ PEDF, with and without serum. Scale bar in $\mathbf{c}=100 \mu \mathrm{m}$. $* * *=$ $P<0.0001$, ANOVA

3 days and $P<0.001$ for $\mathrm{SN}$ versus $\mathrm{pSN}+\mathrm{DC}$ at 7 days and $P<0.0001$ for $\mathrm{SN}$ versus $\mathrm{pSN}+\mathrm{DC}$ at 15 days) in regenerating $\mathrm{pSN}+\mathrm{DC}$ models, compared with the $\mathrm{DC}$ and intact controls, for a period of at least 15 days after injury (Fig. 2c). Western blot and subsequent densitometry confirmed immunohistological changes demonstrating that SN and $\mathrm{pSN}+\mathrm{DC}$ models contained the highest levels of PEDF, with significantly elevated levels $(P<0.001$, ANOVA) present in pSN + DC models (Fig. 2d).

For DRGN to be able to respond to changes in exogenous PEDF, the PEDF-receptor (PEDF-R) must be present in DRGN. Immunostaining for PEDF-R (red) in DRGN was present in DRGN somata in the intact controls and remained unchanged in DC, SN, or pSN + DC models (Fig. 2e). Taken together, these results suggest that PEDF is upregulated in regenerating $\mathrm{SN}$ and $\mathrm{pSN}+\mathrm{DC}$ injury paradigms and remains elevated throughout the first 15 days after injury, albeit more so in pSN + DC models. The PEDF-R is also present in DRGN and so are able to respond to changes in PEDF levels.

\section{PEDF Promotes DRGN Survival in a Dose-Dependent Manner}

To investigate the role of PEDF and its involvement in DRGN survival and neurite outgrowth, a serum-withdrawal DRGN culture model, in the presence of 5-FDU to keep glia involvement to a minimum, was used [5]. In this model, we first confirmed that $60 \%$ DRGN death occurred at 5 days after plating in DMEM without serum (Fig. 3a). DRGN viability at 5 days was then quantified in this serum-withdrawal model after DRGN culture with 0, 50, 100, 200, or 300 ng/ml PEDF peptide and tested for its ability to promote DRGN survival and neurite outgrowth. All doses of PEDF significantly enhanced DRGN viability up to a maximum observed with $100 \mathrm{ng} / \mathrm{ml}$ of PEDF, which promoted $83 \pm 3 \%$ of DRGN survival (Fig. 3b). DRGN survival in the presence of $100 \mathrm{ng} / \mathrm{ml}$ PEDF caused $>50 \%$ more survival than that observed in the absence of PEDF $(P<0.0001)$ and was comparable to survival in the presence of serum (Fig. 3b). Concentrations of PEDF above $100 \mathrm{ng} / \mathrm{ml}$ reduced DRGN survival. These results suggest that PEDF rescues DRGN from death in a serumwithdrawal model. 
a

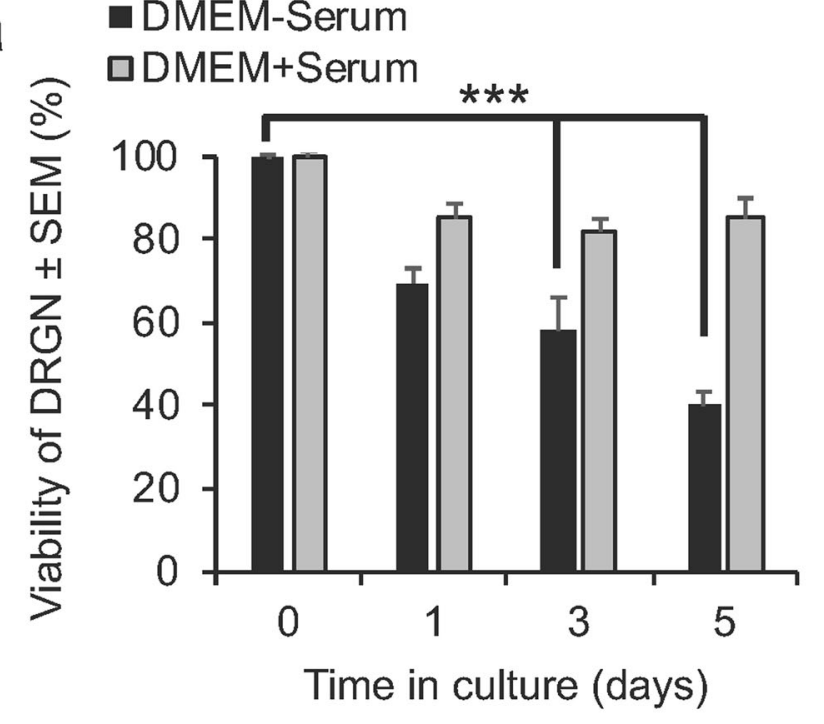

C
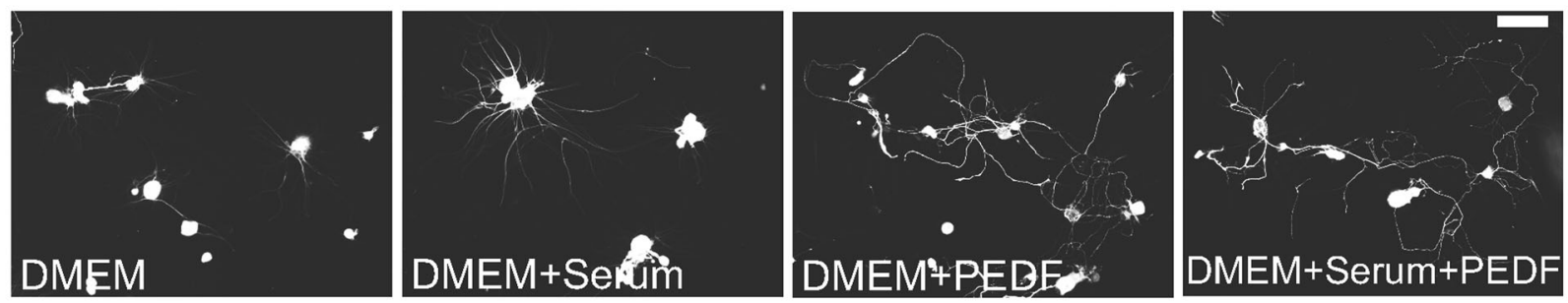

d

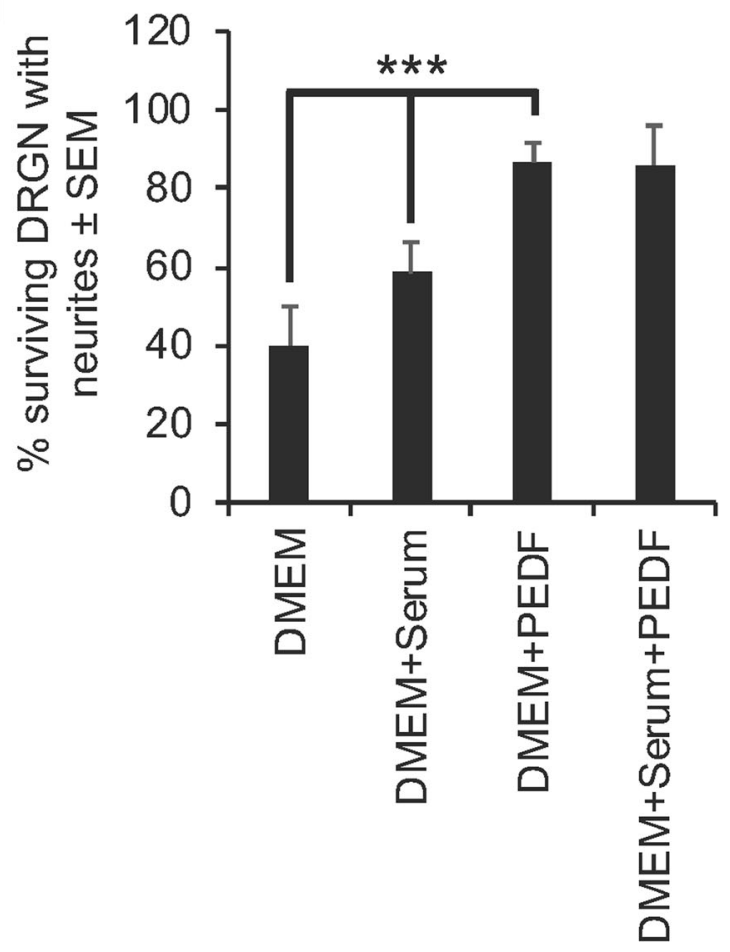

b

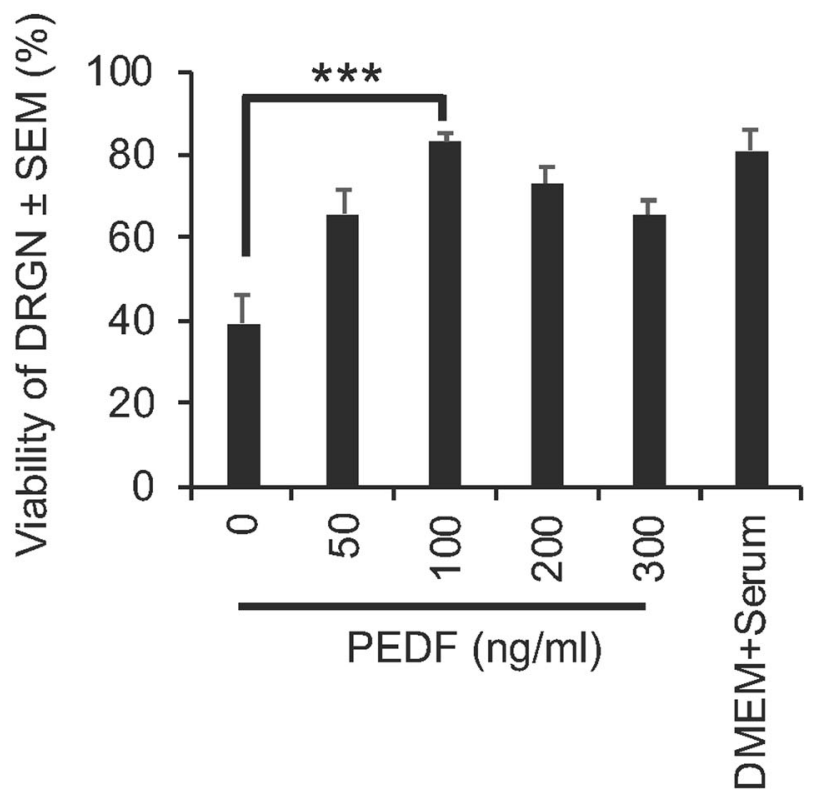

e

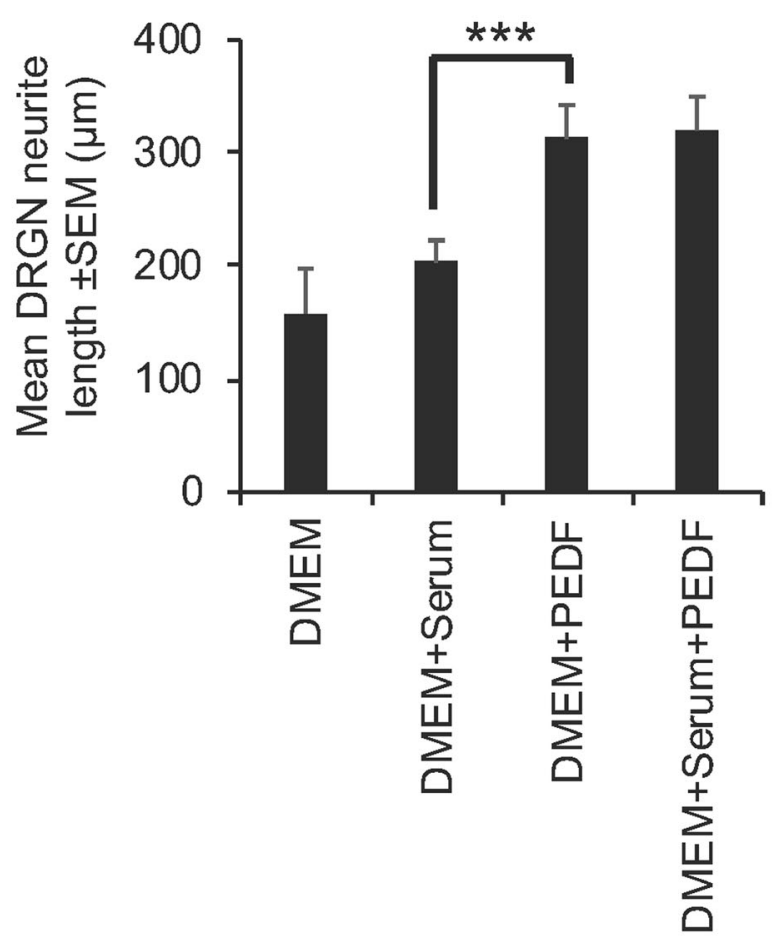




\section{PEDF Is Neuritogenic for DRGN in a Serum Withdrawal Model}

DRGN grown in the presence of $100 \mathrm{ng} / \mathrm{ml}$ of PEDF and $100 \mu \mathrm{g} / \mathrm{ml} \mathrm{CME} \mathrm{showed} \mathrm{enhanced} \mathrm{levels} \mathrm{of} \mathrm{disinhibited}$ neurite outgrowth compared with those grown in DMEM and DMEM + Serum (Fig. 3c). However, there appeared to be no further enhancement in DRGN neurite outgrowth when grown in the presence of DMEM + Serum + PEDF (Fig. 3c). Quantification of the proportion of surviving DRGN with neurites showed that only $40 \pm 10 \%$ and $58 \pm 8 \%$ DRGN grew neurites when cultured in DMEM or DMEM + Serum, respectively (Fig. 3d). However, the proportion of DRGN with neurites increased significantly $(P<0.0001)$ when grown in the presence of either DMEM + PEDF reaching a maximum of $86 \pm 10 \%$, with no further additional enhancement in the proportion of DRGN growing neurites in the presence of DMEM + Serum + PEDF (Fig. 3d). The mean length of the longest neurites was also significantly greater when treated with DMEM + PEDF $(312 \pm 25 \mu \mathrm{m})$ compared with DMEM alone $(158 \pm 40 \mu \mathrm{m})$ or DMEM + serum $(203 \pm 20 \mu \mathrm{m})$ $(P<0.0001)$ (Fig. 3e). Treatment with DMEM + Serum + PEDF did not further potentiate neurite outgrowth when compared with DMEM + PEDF (Fig. 3e). These results demonstrate that $100 \mathrm{ng} / \mathrm{ml}$ PEDF promotes significant DRGN neurite outgrowth.

\section{AAV-Mediated Overexpression of PEDF Promotes DC Axon Regeneration and Functional Recovery}

Intra-DRG injection of AAV-PEDF significantly increased PEDF mRNA levels by $8.6 \pm 0.5$-fold compared with only $0.1 \pm 0.05$-fold mRNA in DC + AAV-Null-treated rats (Supplementary Fig. 1a). The levels of PEDF protein also increased to $687 \pm 29 \mathrm{ng} / \mathrm{mg}$ tissue compared with $11 \pm 7 \mathrm{ng} / \mathrm{mg}$ of tissue in the DC + AAV-Null-treated rats (Supplementary Fig. 1b). These results demonstrated that significant titres of PEDF mRNA and protein were induced in DRGN after intraDRG injection of AAV-PEDF. GAP43 immunohistochemistry showed little or no GAP43 ${ }^{+}$regenerating fibres (green) in DC + AAV-Null-treated rats (Fig. 4a; inset shows high power of boxed region). However, after treatment with AAV-PEDF, significant numbers of $\mathrm{GAP} 43^{+}$regenerating axons were observed in the caudal and rostral segments of the spinal cord (Fig. 4b). Quantification of the number of GAP43 ${ }^{+}$fibres showed that AAV-PEDF promoted the regeneration of $38 \pm$ $5,22 \pm 4,19 \pm 3$, and $17 \pm 3 \%$ of GAP4 $3^{+}$fibres at $0,2,4$, and $6 \mathrm{~mm}$ rostral to the lesion site (Fig. $4 \mathrm{c}$ ).

Electrophysiology showed that the CAP area observed in Sham $(0.71 \pm 0.2 \mathrm{mV} \times \mathrm{ms})$ reduced by $94 \%$ to $0.04 \pm$ $0.05 \mathrm{mV} \times \mathrm{ms}$ in $\mathrm{DC}+\mathrm{AAV}-$ Null-treated rats (Fig. 4d). However, CAP area was significantly improved $(P<0.0001)$ in the AAV-PEDF-treated groups to $64 \%$ of that observed for
Fig. 4 AAV8-mediated overexpression of PEDF promotes DC axon regeneration and improves functional recovery. a Few if any, GAP43 ${ }^{+}$ fibres were present in the caudal or rostral segments of the cord $(*=$ lesion site) in DC + AAV-Null-treated rats. b Numerous GAP43 ${ }^{+}$axons were present in the caudal and rostral segments of the cord after AAV-PEDF treatment. c Quantification of the total number of GAP43 ${ }^{+}$fibres at different distances caudal and rostral to the DC injury site. $\mathbf{d}$ CAP area and $\mathbf{e}$ CAP amplitudes were significantly improved after AAV-PEDF treatment. f Mean sensing time for the tape removal and $\mathbf{g}$ horizontal ladder walking tests both showed improvements in AAV-PEDF-treated rats and no significant differences in both tests by 3 weeks when compared with the Sham controls. Scale bars in $\mathbf{a}$ and $\mathbf{b}=200 \mu \mathrm{m} . *=P<0.05$, ** $=$ $P<0.001$, *** $=P<0.0001$, ANOVA; $+t=P<0.001$, (independent sample $t$ test (DC + AAV-Null versus DC + AAV-PEDF at 2 days); \# = $P<0.01$, generalised linear mixed models; \#\# $=P<0.001$, linear mixed models

the Sham controls (Fig. 4d). The mean CAP amplitude was also significantly reduced in the DC + AAV-Null-treated groups compared with the Sham controls (Fig. 4e). However, significantly larger CAP amplitudes were observed in DC + AAV-PEDF-treated rats at all stimulation intensities, compared with those in the DC + AAV-Null groups $(P<0.001$; Fig. 4e).

The mean sensing time for the tape removal test was between 10 and $26 \mathrm{~s}$ in the Sham-treated controls throughout the 6-week time course (Fig. 4f). However, in the DC + AAVNull-treated groups, sensing time increased significantly to $77 \pm 19 \mathrm{~s}$ at 2 days after DC injury (Fig. 4f). Sensing time decreased over the 6-week period in DC + AAV-Null-treated rats and remained at $36 \pm 3 \mathrm{~s}$ at 6 weeks after DC injury. In contrast, AAV-PEDF-treated rats showed significantly reduced sensing time at 2 days after DC, taking only $38 \pm 4 \mathrm{~s}$ to detect the tape $(P<0.001$, independent sample $t$ test $)$ and were not significantly different with the Sham-treated rats by 3 weeks after DC (Fig. 4f). Over the whole time course, there was a significant reduction in the time taken to sense the adhesive tape in the DC + AAV-PEDF-treated compared with the DC + AAV-Null-treated animals (linear mixed model, $P<0.001)$.

Over a 6-week time course, there was a significant increase in the error rates during the horizontal ladder walking (generalised linear mixed model, $P<0.0011$ ) (Fig. 4g) in DC + AAV-Null-treated compared with DC + AAVPEDF-treated animals. The mean error ratio was significantly lower in DC + AAV-PEDF compared with DC + AAV-Null-treated animals at 2 days after injury $(P<0.001$, independent sample $t$ test $)$ and at 3 weeks after DC injury $(P<0.001$, independent sample $t$ test $)$ by which time the error rates were similar to that of the Sham controls. In the DC + AAV-Null-treated groups, error remained for the full 6-week duration (Fig. 4g). Taken together, these results showed that AAV-PEDF promoted DC axon regeneration that led to improvements in electrophysiological and sensory and locomotor function. 


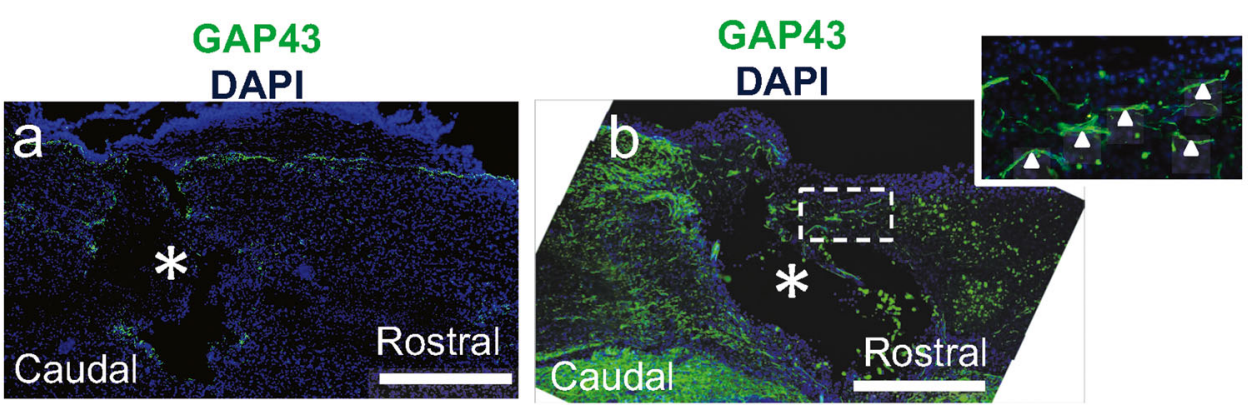

C

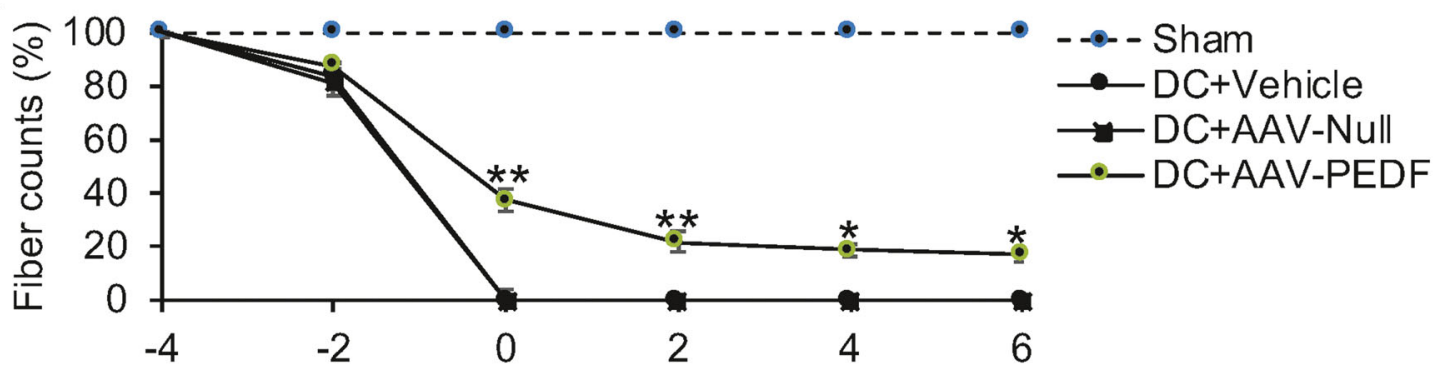

Distance from the lesion $(\mathrm{mm})$

d
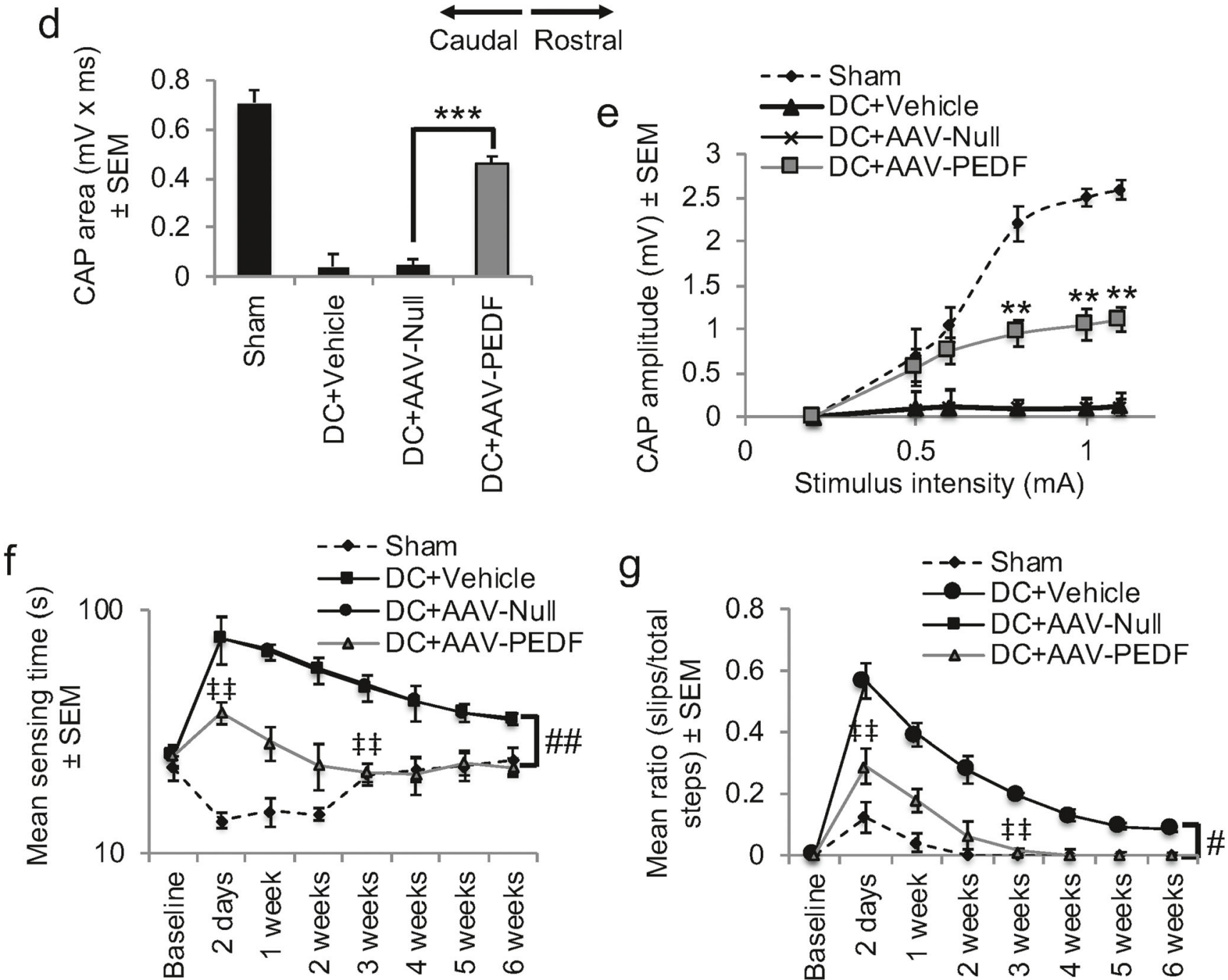


\section{PEI-Mediated Overexpression of PEDF Promotes Similar Functional Recovery as AAV}

In the DC + PEI-PEDF groups, PEDF mRNA was significantly increased to $8.8 \pm 0.8$-fold $(P<0.0001)$ over that observed for either the Sham, DC + Vehicle, or DC + PEI-Null groups (Fig. 5a). Likewise, significant levels of PEDF protein were extracted from DC + PEI-PEDF-treated DRG, with $698 \pm$ $26 \mathrm{ng} / \mathrm{mg}$ compared to only $11 \pm 5$ and $10 \pm 4 \mathrm{ng} / \mathrm{mg}$ of tissue from DC + Vehicle and DC + PEI-Null-treated DRG, respectively (Fig. 5b). These results show that PEI-delivered plasmids overexpressed the expression of PEDF to similar levels in DRG as those observed with AAV8.

Electrophysiology also showed similar improvements in the CAP area (Fig. 5c) and the CAP amplitude (Fig. 5d) in the DC + PEI-PEDF-treated groups as those observed with AAV-PEDF (compare with Fig. 4d, e). For example, the CAP area in the DC + PEI-PEDF-treated groups improved by $69 \%$ of that observed for the Sham controls, whilst the mean CAP amplitudes were also significantly larger in the DC + PEI-PEDF-treated rats at all stimulation intensities, compared with those in the DC + PEI-Null groups $(P<0.001$; Fig. 5d).

PEI-mediated overexpression of PEDF also promoted similar levels of sensory and locomotor functional recovery as AAV. For example, in the DC + PEI-PEDF-treated rats, the mean sensing time was $37 \pm 6 \mathrm{~s}$ at 2 days after DC injury $(P<0.001$, independent sample $t$ test $)$ and sensing times were not significantly different with the Sham-treated rats by 3 weeks after injury (Fig. 5e; $P<0.001$, linear mixed model over the whole time course). The mean error ratios for the horizontal ladder crossing test were also significantly lower in the DC + PEI-PEDF-treated compared with the DC + PEINull-treated rats at 2 days after injury $(P<0.001$, independent sample $t$ test), and by 3 weeks after injury, the error rates were similar with that of the Sham controls (generalised linear mixed model, $P<0.001$ ) (Fig. 5f). Taken together, these results demonstrate that PEI-mediated overexpression of PEDF was just as efficient as AAV in promoting improvements in electrophysiological, sensory, and locomotor function after DC injury in rats.

\section{Addition of Exogenous PEDF Enhances NTF in DRGN Cultures}

To expound a possible mechanism for the neuroprotective and axogenic properties of PEDF, we used dissociated DRGN cultures, grown in the presence of 5-FDU to limit glial proliferation and $\mathrm{CME}$ to mimic the post-injury environment of the degenerating spinal cord to investigate secretion of NTF into the culture medium. We determined that $100 \mathrm{ng} / \mathrm{ml}$ of PEDF caused the secretion of $112 \pm 16,135 \pm 8$, and $102 \pm 12 \mathrm{ng} / \mathrm{ml}$ of BDNF, GDNF, and NGF $(P<0.0001$ DMEM versus
Fig. 5 In vivo-jetPEI-mediated overexpression of PEDF improves similar levels of functional recovery after DC injury as AAV8. DC + PEIPEDF treatment overexpresses PEDF a mRNA and $\mathbf{b}$ protein levels significantly compared with other treatment groups. c CAP area and d CAP amplitudes were significantly improved after DC + PEI-PEDF treatment. e Mean sensing time for the tape removal and $\mathbf{f}$ horizontal ladder walking tests both showed improvements in DC + PEI-PEDF-treated rats and no significant differences in both tests by 3 weeks when compared with the Sham controls. $* *=P<0.001$, $* * *=P<0.0001$, ANOVA; $+t=$ $P<0.001$, independent sample $t$ test (DC + PEI-Null versus DC + PEIPEDF at 2 days); \# $=P<0.01$, generalised linear mixed models; \#\# = $P<0.001$, linear mixed models

DMEM + PEDF) into the culture medium, respectively (Fig. 6a). No ciliary neurotrophic factor (CNTF) nor neurotrophin-3 (NT3) were detected in the culture medium (not shown). Reconstituting the isolated NTF in their relative concentrations in freshly prepared DRGN cultures, grown in the presence of CME, we observed significantly increased DRGN survival (by $>50 \%$; Fig. 6 b), number of surviving DRGN with neurites (i.e. neurite initiation-by $50 \%$; Fig. $6 c$ ), and the mean neurite length (i.e. neurite elongation) from $143 \pm 35 \mu \mathrm{m}$ in DMEM alone to $312 \pm 40 \mu \mathrm{m}$ in DMEM + BDNF + GDNF + NGF-treated cultures (Fig. 6d). The levels of DRGN survival and neurite outgrowth were similar to that observed with DMEM + $100 \mathrm{ng} / \mathrm{ml}$ PEDF treatment (Fig. 6bd). The addition of PEDF to DMEM + BDNF + GDNF + NGF-treated DRG cultures did not further potentiate DRGN survival (Fig. 6b) nor the number of DRGN with neurites (Fig. $6 \mathrm{c})$, but significantly increased $(P<0.0001)$ mean neurite length to $449 \pm 42 \mu \mathrm{m}(P<0.001)$, compared with DRGN neurite outgrowth in DMEM + PEDF or DMEM + BDNF + GDNF + NGF-treated cultures (Fig. 6d, e). These results suggest that PEDF-stimulated NTF, released from DRGN cultures, can promote significant DRGN neurite outgrowth and survival, similar to PEDF-alone-treated cultures, but that the addition of PEDF to BDNF + GDNF + NGF can further potentiate DRGN neurite length (elongation) but not survival nor the number of DRGN with neurites (initiation).

\section{Trk-Dependent Neurotrophins Are Responsible for some of the Neuroprotective and Neuritogenic Properties of PEDF}

In dissociated DRGN cultures, grown in the presence of 5FDU and CME, the neuroprotective effects of PEDF can be partially abrogated by either treatment with K252a, a broadspectrum inhibitor of Trk-dependent neurotrophins or TrkA/B/C-Fc fusion proteins (specific inhibitors of TrkA/B/ C [21]) (Fig. 7a). DRGN survival reduced from $85 \pm 12$ to 64 \pm 5 and $63 \pm 8 \%$ in DMEM + PEDF, DMEM + PEDF + $\mathrm{K} 252 \mathrm{a}$, and DMEM + TrkA/B/C-Fc fusion proteins, respectively. Likewise, K252a and TrkA/B/C-Fc fusion protein treatment only partially suppressed PEDF-stimulated DRGN neurite outgrowth in terms of the number of DRGN with 


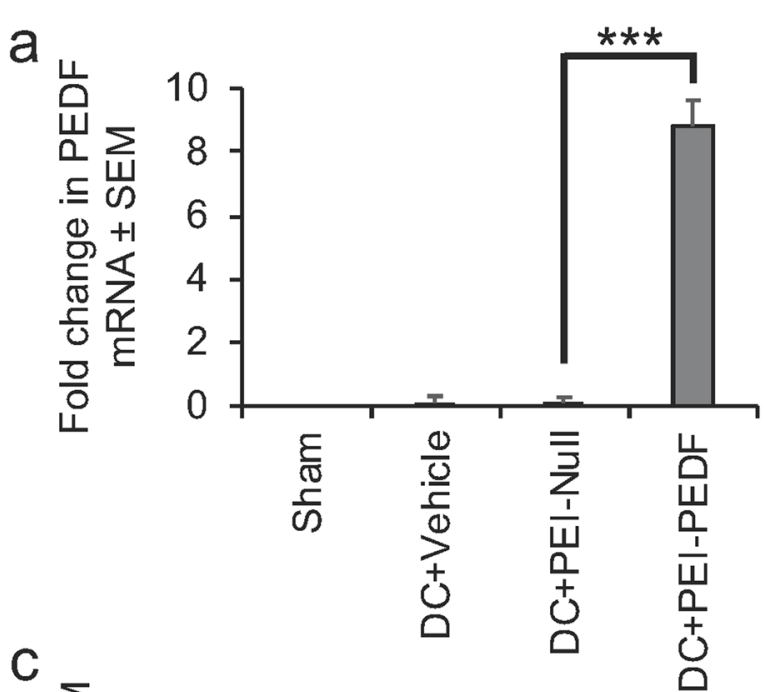

b
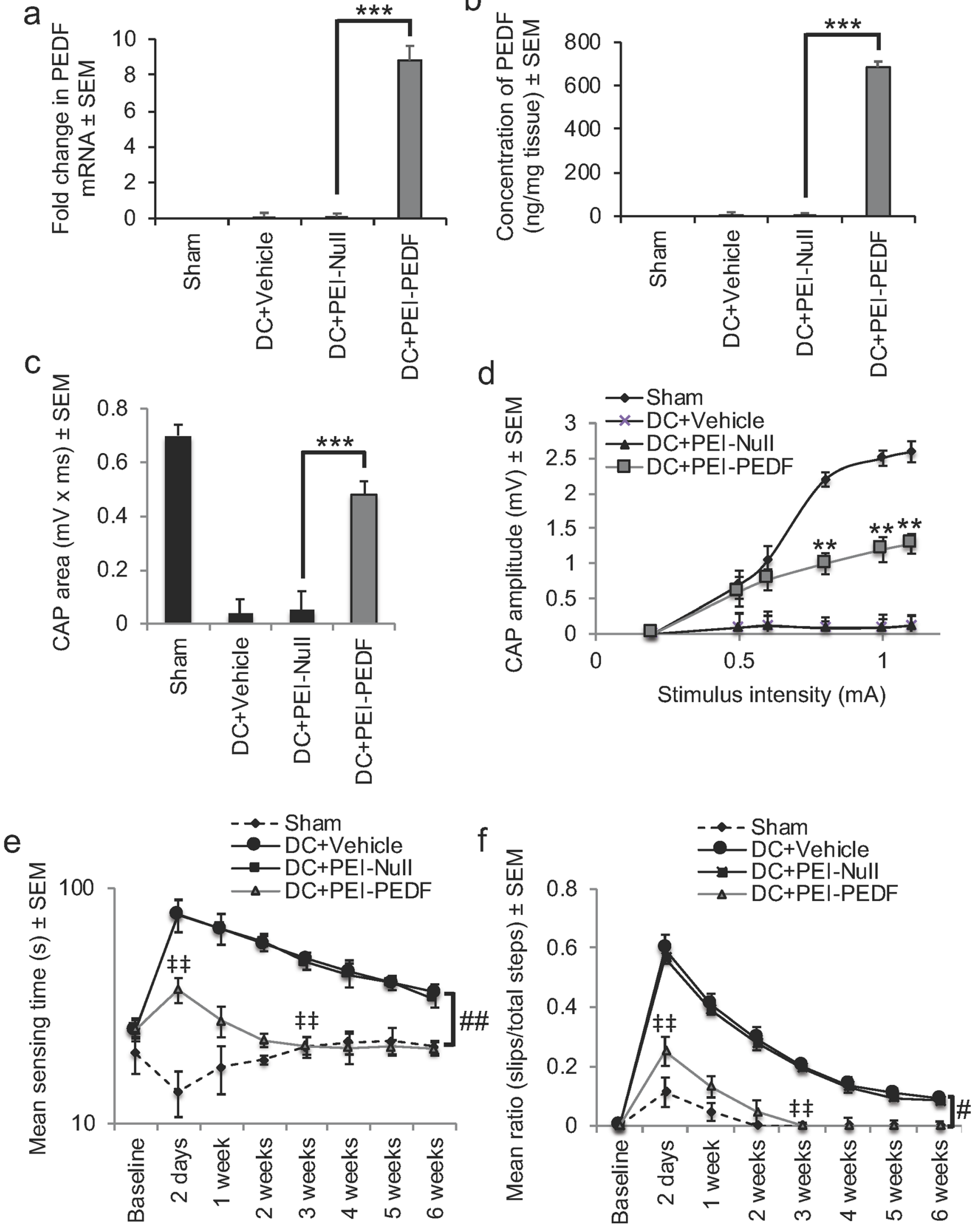
neurites (Fig. 7b) and the mean neurite length (Fig. 7c, d). For example, the mean proportion of DRGN with neurites reduced from $83 \pm 12$ to $44 \pm 8 \%$ and the mean DRGN neurite length reduced from $310 \pm 15$ to $133 \pm 22 \mu \mathrm{m}$ after treatment with $\mathrm{K} 252 \mathrm{a}$ and TrkA/B/C-Fc fusion proteins. These results suggest that DRGN survival and neurite outgrowth are directly and indirectly affected by PEDF and that some of the effects are Trk-dependent.

\section{Discussion}

Here, we have investigated PEDF as a novel target for promoting DRGN survival and neurite outgrowth/axon regeneration and functional recovery in the injured spinal cord. We utilised in vivo regenerating and non-regenerating DRGN models and the in vitro serum-withdrawal model of DRGN cultures, to investigate how the expression of PEDF is modulated in DRGN with contrasting intrinsic growth capacities. We showed that (1) PEDF expression in DRGN in vivo is upregulated in regenerating ( $\mathrm{SN}, \mathrm{pSN}+\mathrm{DC}$ ) models; (2) PEDF-R is expressed in DRGN in vivo; (3) PEDF promotes significant DRGN survival and disinhibited DRGN neurite outgrowth in a serum-withdrawal model and in the presence of inhibitory CME; (4) intra-DRG delivery of AAV-PEDF promoted significant DC axon regeneration and electrophysiological, sensory, and locomotor function; (5) intra-DRG delivery of PEI-PEDF (non-viral vector) promoted similar electrophysiological and functional recovery as AAV8 (viral vector delivery); (6) exposure of DRGN to PEDF in vitro causes the release of significant titres of BDNF, GDNF, and NGF and that these concentrations of NTF significantly enhanced disinhibited DRGN neurite outgrowth; and (7) PEDFinduced DRGN survival and neurite outgrowth are partially suppressed by Trk receptor blockers.

To our knowledge, this is the first study in sensory DRGN to demonstrate a correlation between endogenous upregulation of PEDF and survival/axonal regeneration in DRGN and the ascending DC pathway. PEDF was previously shown to be endogenously upregulated in activated Müller cells and contributed to retinal ganglion cell (RGC) survival in several vision-threatening pathologies [24]. We also showed that PEDF was RGC-neuroprotective and promoted significant RGC axon regeneration in vivo and that PEDF protein was present in RGC, astrocytes and Muller cells in the retina [5, 6]. In contrast, this study has observed that endogenous expression of PEDF was restricted to DRGN and was absent in satellite cells. This study demonstrated both intracellular and extracellular activity for PEDF in regenerating DRGN paradigms.

Results from immunohistochemistry did not identify the presence of an extracellular PEDF-R in DRG satellite cells; PEDF- $\mathrm{R}^{+}$immunostaining was only present around the cell
Fig. 6 PEDF treatment promotes NTF secretion in DRGN cultures. a ELISA was used to detect high levels of BDNF, GDNF, and NGF after exposure of DRGN to $100 \mathrm{ng} / \mathrm{ml}$ PEDF in culture supernatant. b Exposure of DRGN cultures to ELISA-detected levels of BDNF + $\mathrm{GDNF}+\mathrm{NGF}$ and PEDF + BDNF + GDNF + NGF all promotes similarly high levels of DRGN survival and $\mathbf{c}$ the number of DRGN with neurites. However, $\mathbf{d}$ mean neurite length is further potentiated by addition of PEDF compared with DMEM + PEDF-treated cultures. e Representative images to show DRGN neurite outgrowth after treatment with DMEM, DMEM + PEDF, DMEM + BDNF + GDNF + NGF, and $\mathrm{DMEM}+\mathrm{PEDF}+\mathrm{BDNF}+\mathrm{GDNF}+\mathrm{NGF}$. Scale bar in $\mathbf{e}=100 \mu \mathrm{m} . * *=$ $P<0.001, * * *=P<0.0001$, ANOVA

membranes of DRGN. However, the PEDF-R has not been fully characterised, and it is possible that PEDF acts at an alternative, as yet unidentified receptor on satellite cells, which is not detected by the antibody used in this and other previous studies $[25,26]$. PEDF itself, however, was first identified as an NTF secreted by retinal pigment epithelial cells and was originally regarded as an extracellular signalling protein [27]. Concurrent with more recent studies, we detected intracellular PEDF with transient $\mathrm{PEDF}^{+}$nuclear immunostaining $[28,29]$; the nuclear translocation motif of PEDF is mediated by transportin-SR2 [30]. Such an uptake mechanism may be important in mediating the neuroprotective effects of PEDF through further interactions within the nucleus. This would explain our observations that nuclear expression of PEDF is only present in regenerating $(\mathrm{SN}, \mathrm{pSN}+\mathrm{DC})$ models and not in a non-regenerating (DC) model or in the intact control DRGN.

Our study also demonstrated that exogenous PEDF promoted DRGN survival and disinhibited DRGN neurite outgrowth in a serum-withdrawal in vitro model and is the first to show these effects in spinal neurons of adult rats. In addition, AAV-mediated delivery of PEDF to injured DRGN-enhanced DC axon regeneration and improved electrophysical, sensory, and locomotor function in treated rats. Moreover, non-viral vector-mediated plasmid delivery through the use of in vivojetPEI immediately after injury also promoted similar electrophysiological, sensory, and locomotor functional recovery as AAV8-mediated overexpression of PEDF. These results are in accordance with our previous studies in other populations of neurons, where PEDF was neuroprotective and axogenic in RGC both in vitro and in vivo [5, 6].

Through PEDF pathway analysis, we identified an upregulation of NFKB mRNA concurrent with the upregulation of PEDF in regenerating pSN + DC DRGN. Notably, multiple NTF and the anti-apoptotic gene Bcl-2 were also upregulated in this model. As a transcription factor with a nuclear colocalisation signal, activated NFKB mediates upregulation of NTF and anti-apoptotic genes/proteins [31]. NFKB activation is a critical step in preventing neuronal death via a number of pathways throughout the CNS, most notably by mediating the neuroprotective effects of PEDF in cerebellar granule neurons [32]. It is therefore possible that NFKB is responsible for 
a

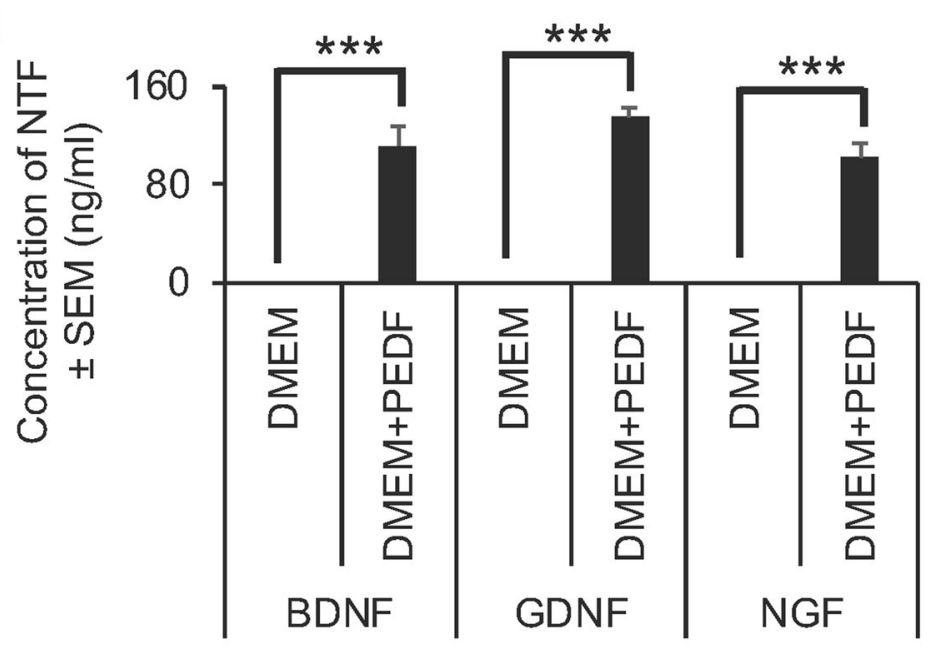

b<smiles>[2H]</smiles>

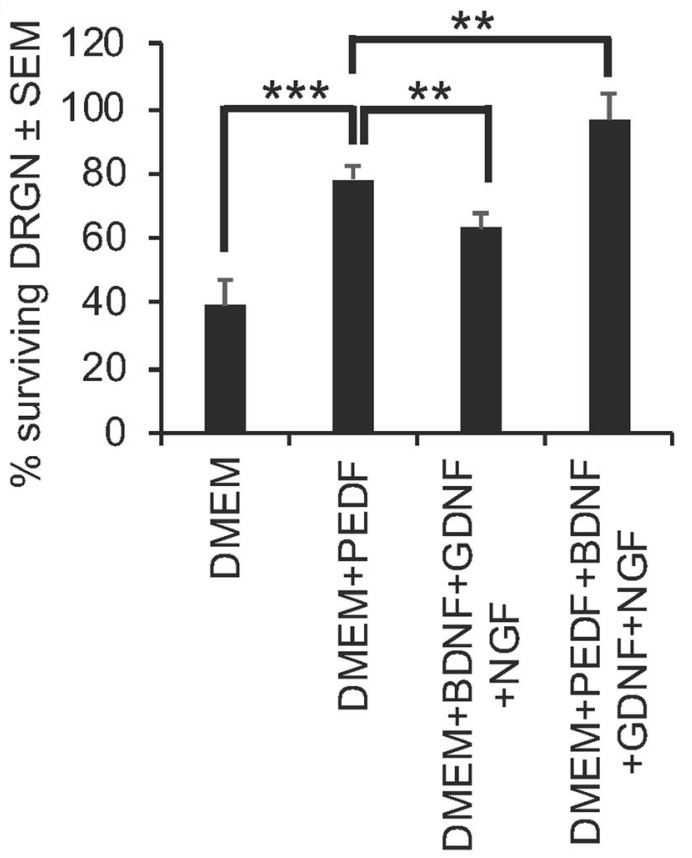

C

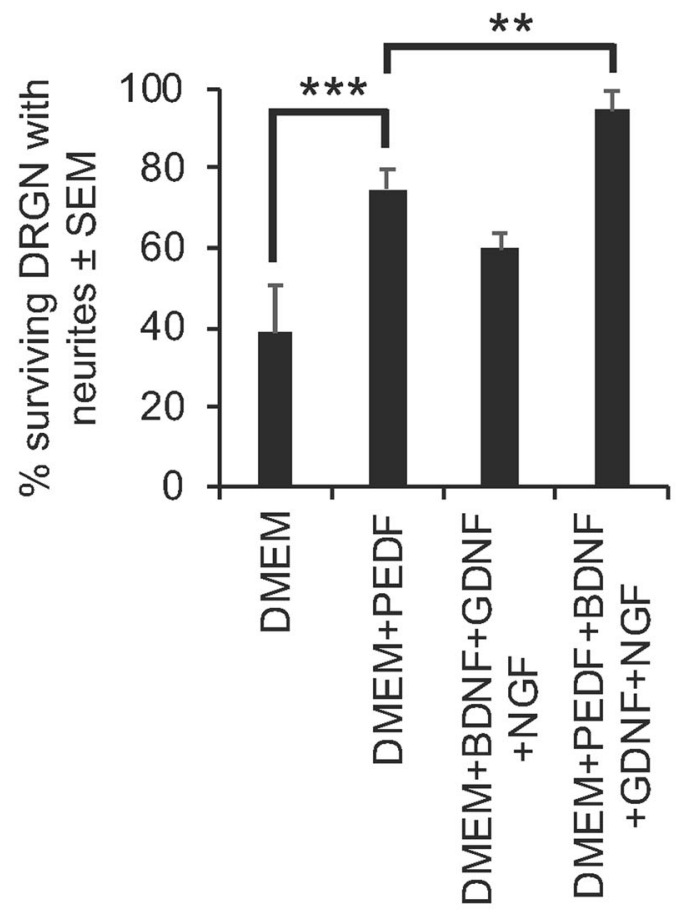

d

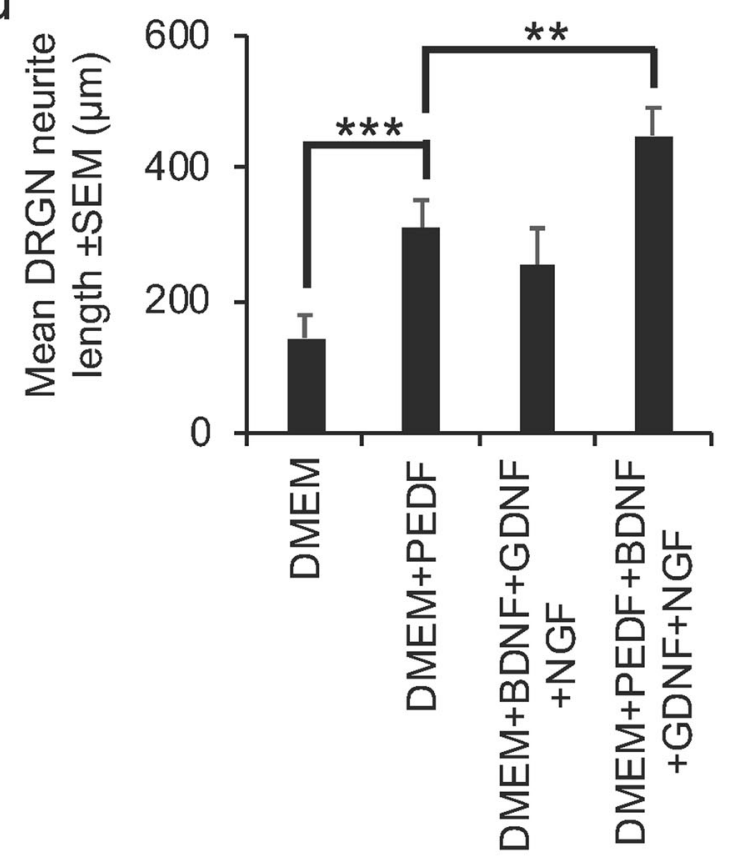

e

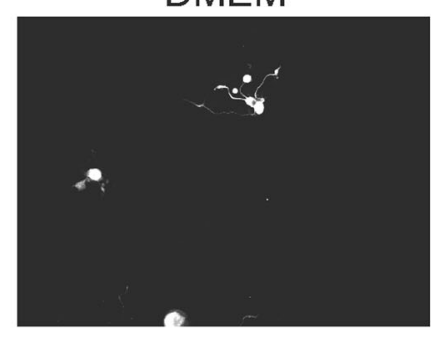

DMEM+BDNF+

GDNF+NGF
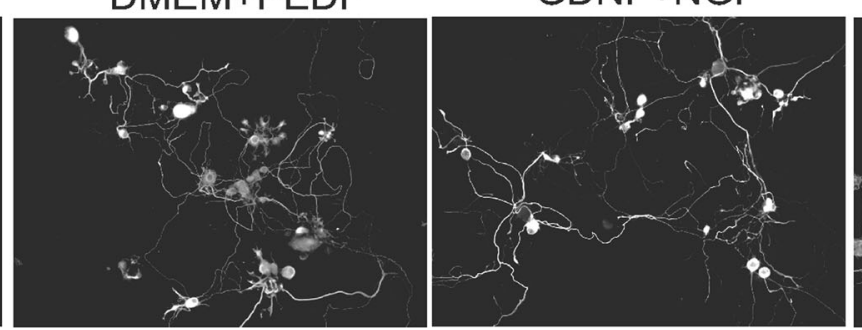

DMEM+PEDF+ $\mathrm{BDNF}+\mathrm{GDNF}+\mathrm{NGF}$

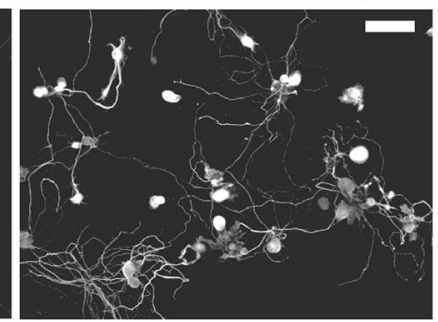



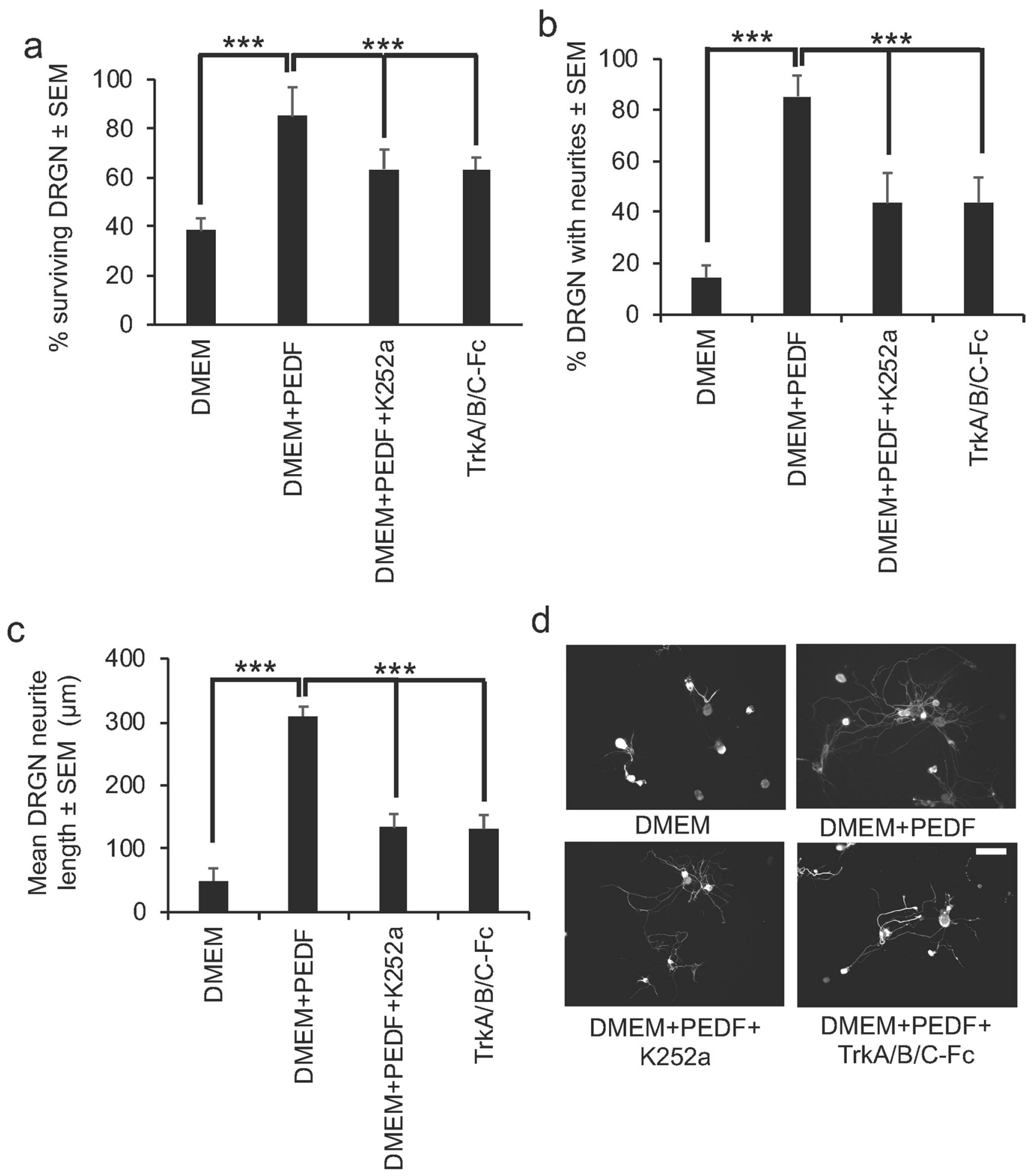

Fig. $7 \mathrm{~K} 252 \mathrm{a}$ and TrkA/B/C-Fc fusion proteins partially abrogate PEDFstimulated DRGN survival and neurite outgrowth. K252a and TrkA/B/CFc fusion proteins significantly reduce a DRGN survival b \% DRGN with neurites and the $\mathbf{c}$ mean neurite length. $\mathbf{d}$ Representative images to

mediating some of the neuroprotective and axogenic effects of PEDF in sensory neurons, through the upregulation of NTF and anti-apoptotic genes/proteins. In this regard, BDNF,

show partial suppression of DRGN neurite outgrowth after treatment with $\mathrm{K} 252 \mathrm{a}$ and TrkA/B/C-Fc fusion proteins. Scale bar in $\mathbf{d}=100 \mu \mathrm{m}$. $* * *=$ $P<0.0001$, ANOVA

GDNF, and NGF were secreted from DRGN in response to PEDF and blockade of Trk receptors, and hence, these NTF do not account for all of the survival and neurite outgrowth- 
promoting effects of PEDF and suggests that PEDF has both direct and indirect effects on DRGN.

Viral vectors, such as AAV8, are commonly used to deliver genes into the CNS but are restricted by insert capacity and 714 days delay in optimal target mRNA expression, limiting their therapeutic effect and their translational potential for use in acute conditions [33-37]. However, non-viral vectors such as in vivo-jetPEI are able to transfect cells in vitro and in vivo, are easy to prepare, stable, and safe; and give immediate therapeutic benefits [38-40]. For example, melanopsin knockdown using in vivo-jetPEI-delivered shRNA was observed within $16 \mathrm{~h}$ after transduction in the eye and lasted for at least 2 months [41]. We used in vivo-jetPEI and monitored $g f p$ expression in DRGN after DC injury and found that in vivojetPEI transduced similar proportions of large diameter DRGN as AAV8, without invoking a non-specific innate immune response $[15,16]$. Given the advantages of in vivo jetPEI over viral vectors, PEDF overexpression using such a non-viral vector presents itself as an exciting therapeutic opportunity to improve functional recovery in spinal cord injury affected patients.

In conclusion, this is the first study to demonstrate that PEDF is an important mediator of DC axon regeneration in the adult mammalian system. We have demonstrated that PEDF is neuroprotective and promotes significant DRGN neurite outgrowth, exhibiting both direct and indirect effects on DRGN. As such, PEDF shows promise to be a potentially novel therapy for neuroprotection and axogenesis after SCI.

Funding Information Funding was provided by the Wellcome Trust (grant no. 092539/Z/10/Z) to Zubair Ahmed and the Wolfson Foundation to Andrew Stevens. The Biotechnology and Biological Sciences Research Council (UK), grant no. G181986, funded the original microarray study.

\section{Compliance with Ethical Standards}

All animal procedures conformed to UK Home Office regulations and local ethics committee guidelines.

Conflict of Interest The authors declare that they have no conflict of interest.

Open Access This article is distributed under the terms of the Creative Commons Attribution 4.0 International License (http:// creativecommons.org/licenses/by/4.0/), which permits unrestricted use, distribution, and reproduction in any medium, provided you give appropriate credit to the original author(s) and the source, provide a link to the Creative Commons license, and indicate if changes were made.

\section{References}

1. Cadotte DW, Fehlings MG (2011) Spinal cord injury: a systematic review of current treatment options. Clin Orthop Relat Res 469(3): 732-741. https://doi.org/10.1007/s11999-010-1674-0
2. Taniwaki T, Becerra SP, Chader GJ, Schwartz JP (1995) Pigment epithelium-derived factor is a survival factor for cerebellar granule cells in culture. J Neurochem 64(6):2509-2517

3. Sanchez A, Tripathy D, Yin X, Luo J, Martinez J, Grammas P (2012) Pigment epithelium-derived factor (PEDF) protects cortical neurons in vitro from oxidant injury by activation of extracellular signal-regulated kinase (ERK) $1 / 2$ and induction of Bcl-2. Neurosci Res 72(1):1-8. https://doi.org/10.1016/j.neures.2011.09.003

4. Houenou LJ, D'Costa AP, Li L, Turgeon VL, Enyadike C, Alberdi E, Becerra SP (1999) Pigment epithelium-derived factor promotes the survival and differentiation of developing spinal motor neurons. J Comp Neurol 412(3):506-514

5. Vigneswara V, Berry M, Logan A, Ahmed Z (2013) Pigment epithelium-derived factor is retinal ganglion cell neuroprotective and axogenic after optic nerve crush injury. Invest Ophthalmol Vis Sci 54(4):2624-2633. https://doi.org/10.1167/iovs.13-11803

6. Vigneswara V, Esmaeili M, Deer L, Berry M, Logan A, Ahmed Z (2015) Eye drop delivery of pigment epithelium-derived factor-34 promotes retinal ganglion cell neuroprotection and axon regeneration. Mol Cell Neurosci 68:212-221. https://doi.org/10.1016/j.mcn. 2015.08.001

7. Chong MS, Woolf CJ, Haque NS, Anderson PN (1999) Axonal regeneration from injured dorsal roots into the spinal cord of adult rats. J Comp Neurol 410(1):42-54

8. Neumann S, Woolf CJ (1999) Regeneration of dorsal column fibers into and beyond the lesion site following adult spinal cord injury. Neuron 23(1):83-91

9. Ahmed Z, Jacques SJ, Berry M, Logan A (2009) Epidermal growth factor receptor inhibitors promote CNS axon growth through offtarget effects on glia. Neurobiol Dis 36(1):142-150. https://doi.org/ 10.1016/j.nbd.2009.07.016

10. Ahmed Z, Douglas MR, Read ML, Berry M, Logan A (2011) Citron kinase regulates axon growth through a pathway that converges on cofilin downstream of RhoA. Neurobiol Dis 41(2):421429. https://doi.org/10.1016/j.nbd.2010.10.012

11. Ahmed Z, Douglas MR, John G, Berry M, Logan A (2013) AMIGO3 is an NgR1/p75 co-receptor signalling axon growth inhibition in the acute phase of adult central nervous system injury. PLoS One 8(4):e61-e878. https://doi.org/10.1371/journal.pone. 0061878

12. Chong MS, Reynolds ML, Irwin N, Coggeshall RE, Emson PC, Benowitz LI, Woolf CJ (1994) GAP-43 expression in primary sensory neurons following central axotomy. J Neurosci 14(7):43754384

13. Ahmed Z, Read ML, Berry M, Logan A (2010) Satellite glia not DRG neurons constitutively activate EGFR but EGFR inactivation is not correlated with axon regeneration. Neurobiol Dis 39(3):292300. https://doi.org/10.1016/j.nbd.2010.04.013

14. Surey S, Berry M, Logan A, Bicknell R, Ahmed Z (2014) Differential cavitation, angiogenesis and wound-healing responses in injured mouse and rat spinal cords. Neuroscience 275:62-80. https://doi.org/10.1016/j.neuroscience.2014.06.003

15. Jacques SJ, Ahmed Z, Forbes A, Douglas MR, Vigenswara V, Berry M, Logan A (2012) AAV8(gfp) preferentially targets large diameter dorsal root ganglion neurones after both intra-dorsal root ganglion and intrathecal injection. Mol Cell Neurosci 49(4):464 474. https://doi.org/10.1016/j.mcn.2012.03.002

16. Almutiri S, Berry M, Logan A, Ahmed Z (2018) Non-viralmediated suppression of AMIGO3 promotes disinhibitied NT3mediated regeneration of spinal dorsal column axons. Sci Rep 8: 10707

17. Ahmed Z, Aslam M, Lorber B, Suggate EL, Berry M, Logan A (2010) Optic nerve and vitreal inflammation are both RGC neuroprotective but only the latter is RGC axogenic. Neurobiol Dis 37(2): 441-454. https://doi.org/10.1016/j.nbd.2009.10.024 
18. Suggate EL, Ahmed Z, Read ML, Eaton-Charnock K, Douglas MR, Gonzalez AM, Berry M, Logan A (2009) Optimisation of siRNA-mediated RhoA silencing in neuronal cultures. Mol Cell Neurosci 40(4):451-462

19. Ahmed Z, Suggate EL, Brown ER, Dent RG, Armstrong SJ, Barrett LB, Berry M, Logan A (2006) Schwann cell-derived factor-induced modulation of the $\mathrm{NgR} / \mathrm{p} 75 \mathrm{NTR} / \mathrm{EGFR}$ axis disinhibits axon growth through CNS myelin in vivo and in vitro. Brain 129(Pt 6): 1517-1533. https://doi.org/10.1093/brain/aw1080

20. Ahmed Z, Mazibrada G, Seabright RJ, Dent RG, Berry M, Logan A (2006) TACE-induced cleavage of NgR and p75NTR in dorsal root ganglion cultures disinhibits outgrowth and promotes branching of neurites in the presence of inhibitory CNS myelin. FASEB J 20(11): 1939-1941. https://doi.org/10.1096/fj.05-5339fje

21. Douglas MR, Morrison KC, Jacques SJ, Leadbeater WE, Gonzalez AM, Berry M, Logan A, Ahmed Z (2009) Off-target effects of epidermal growth factor receptor antagonists mediate retinal ganglion cell disinhibited axon growth. Brain 132 (Pt 11:3102-3121. https://doi.org/10.1093/brain/awp240

22. Ahmed Z, Bansal D, Tizzard K, Surey S, Esmaeili M, Gonzalez AM, Berry M, Logan A (2014) Decorin blocks scarring and cystic cavitation in acute and induces scar dissolution in chronic spinal cord wounds. Neurobiol Dis 64:163-176. https://doi.org/10.1016/j. nbd.2013.12.008

23. Fagoe ND, Attwell CL, Eggers R, Tuinenbreijer L, Kouwenhoven D, Verhaagen J, Mason MR (2016) Evaluation of five tests for sensitivity to functional deficits following cervical or thoracic dorsal column transection in the rat. PLoS One 11(3):e0150141. https://doi.org/10.1371/journal.pone.0150141

24. Unterlauft JD, Eichler W, Kuhne K, Yang XM, Yafai Y, Wiedemann P, Reichenbach A, Claudepierre T (2012) Pigment epithelium-derived factor released by Muller glial cells exerts neuroprotective effects on retinal ganglion cells. Neurochem Res 37(7):1524-1533. https://doi.org/10.1007/s11064-012-0747-8

25. Filleur S, Nelius T, de Riese W, Kennedy RC (2009) Characterization of PEDF: A multi-functional serpin family protein. J Cell Biochem 106(5):769-775. https://doi.org/10.1002/jcb. 22072

26. Subramanian P, Rapp M, Becerra SP (2012) Identification of pigment epithelium-derived factor receptor (PEDF-R) antibody epitopes. Adv Exp Med Biol 723:799-805. https://doi.org/10.1007/ 978-1-4614-0631-0 102

27. Steele FR, Chader GJ, Johnson LV, Tombran-Tink J (1993) Pigment epithelium-derived factor: neurotrophic activity and identification as a member of the serine protease inhibitor gene family. Proc Natl Acad Sci U S A 90(4):1526-1530

28. Tombran-Tink J, Shivaram SM, Chader GJ, Johnson LV, Bok D (1995) Expression, secretion, and age-related downregulation of pigment epithelium-derived factor, a serpin with neurotrophic activity. J Neurosci 15(7 Pt 1):4992-5003

29. Karakousis PC, John SK, Behling KC, Surace EM, Smith JE, Hendrickson A, Tang WX, Bennett J et al (2001) Localization of pigment epithelium derived factor (PEDF) in developing and adult human ocular tissues. Mol Vis 7:154-163

30. Anguissola S, McCormack WJ, Morrin MA, Higgins WJ, Fox DM, Worrall DM (2011) Pigment epithelium-derived factor (PEDF) interacts with transportin SR2, and active nuclear import is facilitated by a novel nuclear localization motif. PLoS One 6(10):e26234. https://doi.org/10.1371/journal.pone.0026234

31. Mattson MP (2005) NF-kappaB in the survival and plasticity of neurons. Neurochem Res 30(6-7):883-893. https://doi.org/10. 1007/s11064-005-6961-x

32. Yabe T, Wilson D, Schwartz JP (2001) NFkappaB activation is required for the neuroprotective effects of pigment epitheliumderived factor (PEDF) on cerebellar granule neurons. J Biol Chem 276(46):43313-43319. https://doi.org/10.1074/jbc.M107831200

33. Davidson BL, Breakefield XO (2003) Viral vectors for gene delivery to the nervous system. Nat Rev Neurosci 4(5):353-364. https:// doi.org/10.1038/nrn1104

34. Raoul C, Barker SD, Aebischer P (2006) Viral-based modelling and correction of neurodegenerative diseases by RNA interference. Gene Ther 13(6):487-495. https://doi.org/10.1038/sj.gt.3302690

35. Miyagoe-Suzuki Y, Takeda S (2010) Gene therapy for muscle disease. Exp Cell Res 316(18):3087-3092. https://doi.org/10.1016/j. yexcr.2010.05.022

36. Mason MR, Ehlert EM, Eggers R, Pool CW, Hermening S, Huseinovic A, Timmermans E, Blits B et al (2010) Comparison of AAV serotypes for gene delivery to dorsal root ganglion neurons. Mol Ther 18(4):715-724. https://doi.org/10.1038/mt.2010.19

37. McCarty DM, Monahan PE, Samulski RJ (2001) Selfcomplementary recombinant adeno-associated virus (scAAV) vectors promote efficient transduction independently of DNA synthesis. Gene Ther 8(16):1248-1254. https://doi.org/10.1038/sj.gt. 3301514

38. Boussif O, Lezoualc'h F, Zanta MA, Mergny MD, Scherman D, Demeneix B, Behr JP (1995) A versatile vector for gene and oligonucleotide transfer into cells in culture and in vivo: polyethylenimine. Proc Natl Acad Sci U S A 92(16):7297-7301

39. Wiseman JW, Goddard CA, McLelland D, Colledge WH (2003) A comparison of linear and branched polyethylenimine (PEI) with DCChol/DOPE liposomes for gene delivery to epithelial cells in vitro and in vivo. Gene Ther 10(19):1654-1662. https://doi.org/ 10.1038/sj.gt. 3302050

40. Lungwitz U, Breunig M, Blunk T, Gopferich A (2005) Polyethylenimine-based non-viral gene delivery systems. Eur J Pharm Biopharm 60(2):247-266. https://doi.org/10.1016/j.ejpb. 2004.11.011

41. Liao HW, Yau KW (2007) In vivo gene delivery in the retina using polyethylenimine. Biotechniques 42(3):285-286, 288. https://doi. org/10.2144/000112404

Publisher's Note Springer Nature remains neutral with regard to jurisdictional claims in published maps and institutional affiliations. 